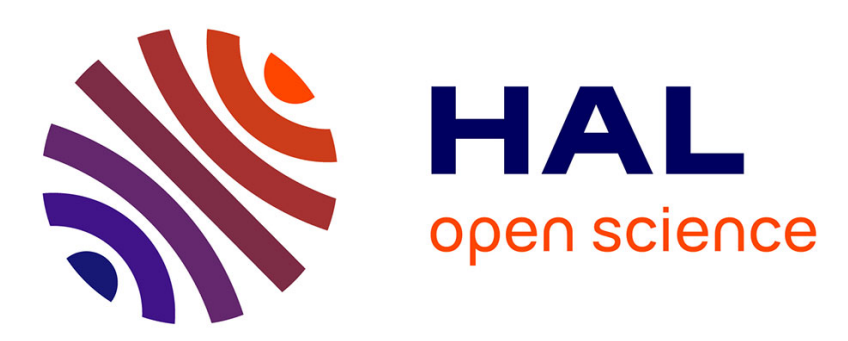

\title{
An investigation of the mechanisms of sound generation in initially laminar subsonic jets using the Goldstein acoustic analogy
}

\author{
Sergey Karabasov, Christophe Bogey, Tom Hynes
}

\section{- To cite this version:}

Sergey Karabasov, Christophe Bogey, Tom Hynes. An investigation of the mechanisms of sound generation in initially laminar subsonic jets using the Goldstein acoustic analogy. Journal of Fluid Mechanics, 2013, 714, pp.24-57. 10.1017/jfm.2012.448 . hal-00931432

\section{HAL Id: hal-00931432 \\ https://hal.science/hal-00931432}

Submitted on 17 Jun 2014

HAL is a multi-disciplinary open access archive for the deposit and dissemination of scientific research documents, whether they are published or not. The documents may come from teaching and research institutions in France or abroad, or from public or private research centers.
L'archive ouverte pluridisciplinaire HAL, est destinée au dépôt et à la diffusion de documents scientifiques de niveau recherche, publiés ou non, émanant des établissements d'enseignement et de recherche français ou étrangers, des laboratoires publics ou privés. 


\title{
An investigation of the mechanisms of sound generation in initially laminar subsonic jets using the Goldstein acoustic analogy
}

\author{
Sergey Karabasov ${ }^{1} \dagger$, Christophe Bogey ${ }^{2}$ and Tom Hynes ${ }^{1}$ \\ ${ }^{1}$ Whittle Laboratory, Department of Engineering, University of Cambridge, Cambridge CB3 ODY, UK \\ ${ }^{2}$ Laboratoire de Mécanique des Fluides et d'Acoustique, UMR CNRS 5509, Ecole Centrale de Lyon, \\ Université de Lyon, 69134 Ecully, France
}

(Received 19 December 2011; revised 24 July 2012; accepted 10 September 2012)

The details of the sound sources within two initially laminar subsonic jets corresponding to different inflow conditions, one fully laminar and the other with nozzle boundary layer forcing, are studied using the Goldstein acoustic analogy. The statistics of the acoustic analogy equivalent sources are generated from large eddy simulations (LES) and issues associated with the range of validity of these are explored. The predicted comparative importance of various source terms confirms the results of previous studies, with the exception of one extra term that involves the longitudinal and the lateral source components. Agreement within $1 \mathrm{~dB}$ over the frequency range from Strouhal number $S t=0.8$ to $S t=6$, which was shown to best correspond to the validity range of the acoustic source model for the LES data available, is found between noise predictions using the acoustic analogy model and those of a previously conducted LES-ILEE (isotropic linearized Euler equation) control surface method for the nominally laminar inflow jet case. The acoustic analogy is used to determine the source length scales which contribute to noise at different radiated angles to the jet, how they are distributed and how they change with change of inflow conditions. For the vortex pairing frequency, using a new acoustic source decomposition technique based on the correlation length scale, two types of noise sources are found. One source, which corresponds to the peak source amplitude, is located at the upstream end of the jet and is associated with the vortex pairing. The other one, which is the dominant source, is associated with jet mixing at the end of the jet potential core. It is also shown that boundary layer forcing leads to a reduction in the contribution to the noise of the large source length scales in comparison with the fully laminar case.

Key words: acoustics, aeroacoustics, jet noise

\section{Introduction}

It is well known that, for transitional flows, the properties of the upstream boundary layer strongly affect the downstream flow development. For transitional jet flows, in

$\dagger$ Present address: School of Engineering and Materials Science, Queen Mary University of London, Mile End Road, London E1 4NS, UK. Email address for correspondence: s.karabasov@qmul.ac.uk 
particular, the properties of the nozzle-exit boundary layer have been shown to strongly influence the jet development and its acoustics, especially for initially laminar jet flow. For example, in the works of Maestrello \& McDaid (1971), Grosche (1974) and Hill, Jenkins \& Gilbert (1976) it is demonstrated that the variation in nozzle boundary layer parameters, such as the momentum thickness and the level of velocity fluctuations, strongly modifies the entire flow mixing process and, as a byproduct, changes the radiated sound field. According to the Crighton (1981) analysis of experimental data, jets exhibit an initially laminar state for Reynolds numbers up to around $10^{5}$ based on the nozzle diameter. In a series of experiments with initially laminar jets of Reynolds number of $2.5 \times 10^{5}$, Zaman $(1985 a, b)$ show that the organized features of laminar jets, such as the roll-up and pairing of coherent vortex structures, dominate over the random turbulent jet mixing effects within the first few jet diameters from the nozzle exit. For far-field sound, these features become effective noise sources that lead to additional peaks in the noise spectra. The additional acoustic sources strongly depend on the nozzle-exit boundary layer state: their intensity diminishes with a decrease of the boundary layer thickness and they can be removed by tripping the boundary layer. Taking into account the differences in inflow boundary conditions for jet noise may also have an important practical aspect for noise measurements obtained in different test facilities. Most recently, by conducting a set of careful experiments, Zaman (2011) shows that variation of the nozzle exit conditions can lead to a few decibels (dB) difference in sound pressure levels measured for otherwise similar high-speed subsonic jets.

The experimental investigation of the effect of initial jet conditions on acoustics is a challenge: despite a large body of literature on jet noise since the 1970s there are only relatively few studies where the nozzle-exit conditions are well documented (such as in Bridges \& Hussain 1987), since usually the inflow parameters cannot be changed independently. Computational modelling can be a viable complementary tool for analysis of the effect of inflow conditions (e.g. Stanley \& Sarkar 2000; Bogey \& Bailly 2005). There is, however, a need to consider more carefully the effect of computational resolution limitations on the numerical prediction results when attempting to resolve thin boundary layers typical of high -Reynolds-number jets (e.g. Colonius \& Lele 2004).

Recently, Bogey \& Bailly (2010) partially resolved this issue with the use of large eddy simulation (LES) combining a low-dissipation algorithm with relaxation filtering (Bogey \& Bailly 2009) and a set of high-resolution grids with progressive refinement for a canonical isothermal jet case. In their study several isothermal axisymmetric jets were considered to investigate the effects of the nozzle-exit conditions on the jet aerodynamics and acoustics. The differing nozzle-exit conditions, which were inlet conditions for the jet calculation, led to noticeably different shear layer behaviour. This, in turn, led to differences in the far-field sound, as predicted using an approach in which the linearized Euler equations (LEE) were coupled to the LES solution at an open cylindrical surface outside of the jet. The study of Bogey \& Bailly (2010), among other things, shows how slightly disturbing the nozzle-exit boundary layer significantly reduces the peaks in the noise spectra associated with the vortex roll-up and pairing frequencies. However, that study also leaves some open questions: what are the characteristics of the effective mechanisms of noise for different inflow jet conditions and, most importantly, how do the differences in the inflow conditions drive the changes in turbulent jet mixing that ultimately translate to jet mixing noise?

There is no general agreement, and perhaps it is a matter of philosophical debate, about what are the 'true' sources of sound for fully nonlinear flows and whether it is 
possible, therefore, to fully extract them. In this paper, however, we will try our best to achieve this goal by using the language of acoustic analogy (Lighthill 1952) to obtain a useful definition of the source mechanisms that is both insightful and tractable. Our method of analysis will be based on a model in which the unsteady flow is accurately decomposed into nonlinear sources and linear propagation effects. For this decomposition, we will use one of the most advanced acoustic analogy formulations currently available (Goldstein 2002, 2003). We will apply this analysis method to the high-resolution LES data of Bogey \& Bailly (2010). The measure of success of our acoustic modelling will be based on the validation of its results for far-field noise spectra against the reference solutions of Bogey \& Bailly for different observer angles. The reference solutions were obtained from a first-principle large-scale calculation and used no modelling, hence can be regarded, in this context, as the 'true solution'. From this validation exercise, we will estimate the applicability range of the acoustic model. For the cases when the acoustic analogy predictions can be considered to be close enough to the 'truth' (e.g. within $1 \mathrm{~dB}$ ), a definitive conclusion about the effective sources of sound that are captured by the current acoustic analogy model will be drawn.

Jet noise modelling has been an active topic of research for 60 years since the acoustic analogy approach of Lighthill (1952). In the Lighthill theory the Navier-Stokes $(\mathrm{N}-\mathrm{S})$ equations are rearranged exactly to form a linear wave equation for the fluctuation in density

$$
\left(\frac{1}{c_{0}^{2}} \frac{\partial^{2}}{\partial t^{2}}-\frac{\partial^{2}}{\partial x_{i} \partial x_{i}}\right)\left(\rho-\rho_{0}\right)=q(\boldsymbol{x}, t),
$$

where the source on the right-hand side effectively includes all the rest of the $\mathrm{N}-\mathrm{S}$ terms $q(\boldsymbol{x}, t)=\left(\partial^{2} / \partial x_{i} \partial x_{j}\right)\left(\rho v_{i} v_{j}+\left(\left(p-p_{0}\right)-c_{0}^{2}\left(\rho-\rho_{0}\right)\right) \delta_{i j}-\sigma_{i j}\right)=\left(\partial^{2} / \partial x_{i} \partial x_{j}\right) T_{i j}$. Here $v_{i}, \rho, p, c, \sigma_{i j}$ are the Cartesian velocity components, density, pressure, sound speed, viscous stress tensor, respectively, and sub-index 0 indicates the undisturbed far-field flow parameters and $\delta_{i j}$ is the Kronecker delta. A weakness of this formulation is that all acoustic propagation effects associated with the jet are incorporated into $q$ and must effectively be neglected. Nevertheless, by using a number of additional assumptions for high-speed subsonic jets, such as neglecting the effect of viscous dissipation and considering turbulent eddies to be acoustically compact, the celebrated $v^{8}$ Lighthill law is obtained (e.g. Howe 2003). Despite the assumptions used in its derivation that are formally correct only for low-Mach-number flows, the scaling of the acoustic energy with the eighth power of the nozzle-exit jet velocity works surprisingly well in many high-subsonic-Mach-number isothermal jet experiments (Viswanathan 2009).

Since Lighthill, further improvements to the original approach have been developed by Lilley (1958), Ffowcs Williams (1963), Ribner (1964), Goldstein \& Rosenbaum (1973) and Tester \& Morfey (1976), to name but a few. These formulations differ by how the problem is decomposed into an acoustic source description and the equations used to describe propagation effects. For example, one of the early approaches based on Lilley's (1972) equation, which is still used in modern noise prediction codes (Khavaran, Bridges \& Georgiadis 2005), explicitly accounts for sound interaction with a simplified jet mean flow. More discussion on acoustic analogy methods can be also found in Morris \& Farassat (2002), Tam et al. (2008), Michel (2009), Morris (2009), Karabasov (2010) and Goldstein (2011).

For the purpose of describing the acoustic analogy used in the present paper, it is appropriate to discuss the following works. Tam \& Auriault (1998) considered 
the Euler equations linearized about a general steady Euler equation solution for the study of sound refraction from a point source embedded in a jet. For a unidirectional mean flow, which is also used by Lilley (1958), Tam \& Auriault (1999) solved the linearized Euler equations with a simplified isotropic turbulent source which was based on a (steady) Reynolds-averaged Navier-Stokes (RANS) solution fitted with empirical coefficients. Goldstein (2002) exactly rearranged the N-S equations to express the farfield acoustic spectrum as a product of a linearized Euler propagator that accounts for complete sound-mean flow interaction effects and a source term, that for isothermal jets, reduces to a generalized fluctuating Reynolds stress tensor. The latter, under conditions of statistical stationarity of jet turbulence, involves fourth-order correlations of turbulence properties. Goldstein (2003) further rearranged this acoustic analogy formulation to a more convenient form for theoretical analysis.

It should be remarked that, in common with the classical Lighthill acoustic analogy, the Goldstein formulation also assumes a one-way coupling between the hydrodynamic and acoustic fields since the generalized fluctuating Reynolds stress does not include an explicit dependence on the acoustic variable. In contrast to the Lighthill equation, the Goldstein formulation allows an explicit treatment of rather complex effects of sound/non-uniform mean flow interference that mimic the dispersion properties of the original Navier-Stokes equations (e.g. see the discussion in Karabasov et al. 2010). The Goldstein formulation also presents a more consistent decomposition of the flow field into nonlinear source and linear propagation with an apparently tighter causal dependence of the radiated sound on the source. For example, by conducting a direct numerical simulation (DNS) of unsteady Navier-Stokes equations for a twodimensional shear layer problem, Samanta et al. (2006) showed that different acoustic analogy models have different sensitivities to errors in the source. They found that those models which explicitly account for more propagation effects, such as the Goldstein $(2002,2003)$ generalized acoustic analogy, tend to be more accurate.

Goldstein \& Leib (2008) used the Goldstein analogy approach to obtain a closedform formula for the far-field sound spectrum using a number of simplifying assumptions about the jet mean flow and properties of the turbulence. A further development of the Goldstein method is used by Karabasov et al. (2010) to capture numerically the jet mean flow-sound interaction effects by solving linearized Euler equations about the complete jet mean flow and by modelling the jet source statistics through a combination of processed LES data and the prediction of length and time scales computed from a separate RANS calculation. That work demonstrated the importance of accurately modelling the mean flow/sound interaction, highlighting the dominance of a few of the fourth-order correlations and resulted in a computational model for sound predictions that showed encouraging agreement with measurements, agreeing to within $2 \mathrm{~dB}$ for a range of frequencies and observer angles to the jet.

The work described in the current paper, which is an expanded version of the recent AIAA conference paper of the authors (Karabasov et al. 2011), attempts to apply the formulation described in Karabasov et al. (2010) to two of the jets corresponding to different inflow conditions for which calculations were performed by Bogey \& Bailly (2010). One aim of this paper is to perform a more detailed test of the assumptions underlying the scaling of terms using the acoustic analogy method described in Karabasov et al. (2010). The second aim is to examine whether the formulation described in Karabasov et al. (2010) is robust enough to distinguish between the different cases described in Bogey \& Bailly (2010) in terms of the predicted far-field sound. In order to avoid the difficulties of applying RANS methods to initially laminar jets and, more importantly, to avoid issues of inconsistencies between any RANS and 
LES mean flows, no RANS calculation will be used in this study. Instead, all mean flow properties and turbulence statistics will be extracted from the LES calculations under the assumption of statistical stationarity. As in the previous study of Karabasov et al. (2010), the sensitivity of the current model to its individual components will be investigated. The final aim is to attempt to explain further how the changes in nozzle-exit conditions produce the calculated effect on far-field sound through a detailed numerical imaging of acoustic analogy noise sources in each case. In $\S 2$ the two jets cases and the available LES are described. In $\S 3$ the general framework of the Goldstein acoustic analogy is introduced. In $\S 4$, the acoustic processing procedure for the LES data is described and the resulting characteristics of the acoustic analogy equivalent source are discussed. The acoustic source characteristics are used for sound prediction in $\S 5$ where the far-field predictions are presented and compared with the reference solutions of Bogey \& Bailly (2010). A sensitivity study to the various components of the model is also performed. Finally, $\S 6$ is devoted to a detailed investigation of effective acoustic sources for the two jet cases considered by attempting to clarify which spatial source scales and which frequencies contribute most to noise, where precisely in the jet these acoustic source scales are located, and also to suggest plausible fluid dynamic mechanisms that create these effective sound sources.

\section{Jet properties and LES details}

The jets studied by Bogey \& Bailly (2010) are isothermal, have a Mach number of 0.9 and a Reynolds number of $10^{5}$ based on nozzle diameter. They issue from a short pipe nozzle (length $/ r_{0}=1.1$, where $r_{0}$ is the nozzle-exit radius) with Blasius boundary layer velocity profiles of varying thickness imposed at nozzle inlet. That paper discussed in detail a variety of initial boundary layer thicknesses and two cases for which a random forcing was applied, within a section of the nozzle boundary layer, in order to generate disturbances at the nozzle exit. In general, the predictions of the effects of the nozzle-exit boundary layer thickness and of tripping the boundary layer on noise were in good agreement with the laminar jet noise experiments reported by Zaman \& Hussain (1980) and Zaman (1985a) (figure 1), as well as with more recent numerical studies by Bogey, Marsden \& Bailly $(2011 a, b)$.

The two particular jets considered in this paper correspond to those with a shear layer thickness of $0.05 r_{0}$ (momentum thickness of $0.0056 r_{0}$ ). One of them has a fully laminar inflow condition (referred to as 'untripped'), with any unsteadiness generated as a self-sustaining part of the LES solution after the withdrawal of an initial short period of seeded unsteadiness. The other has a random forcing unsteady pressure of amplitude $2000 \mathrm{~Pa}$. The forcing is imposed within the boundary layers between $z=-0.4 r_{0}$ and $z=-0.2 r_{0}$. Details of the numerical implementation can be found in Bogey \& Bailly (2010). This corresponds to what Zaman (1985a) described as 'nominally laminar' and what we shall refer to as 'tripped'. Both jets have low initial peak root-mean-square (r.m.s.) axial velocity values: below $1 \%$ of the jet velocity for the untripped case, and around $1.9 \%$ for the tripped. The potential core length of the untripped jet is 7 jet diameters and that of the tripped one is 9. As shown by Bogey $\&$ Bailly, this difference in the inflow conditions, that is negligible in comparison to mean flow quantities, can lead to a $5-10 \mathrm{~dB}$ change in the peak sound levels. A major acoustic mechanism in the two jets is thought to be associated with vortex pairing, which Bogey \& Bailly identified as having a characteristic frequency $S t=2.16$ and $S t=1.61$ for the tripped and untripped jets respectively, where the Strouhal number $S t$ is defined using frequency in radians and jet diameter. 
(a)

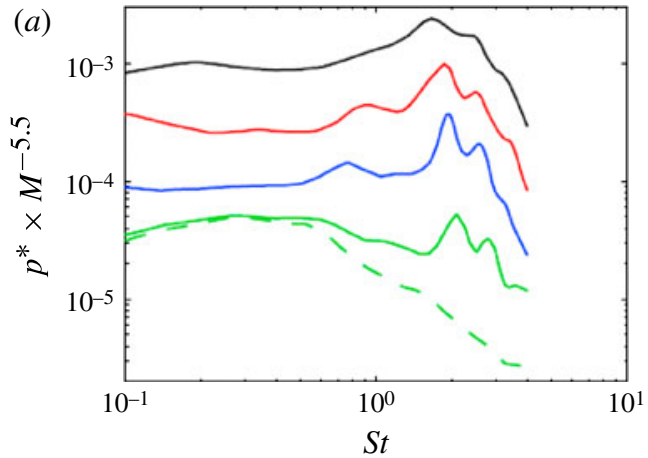

$\delta_{\theta} / r_{0}=-0.8 \%$

$\delta_{\theta} / r_{0}=-0.6 \%$

--- Tripped jet

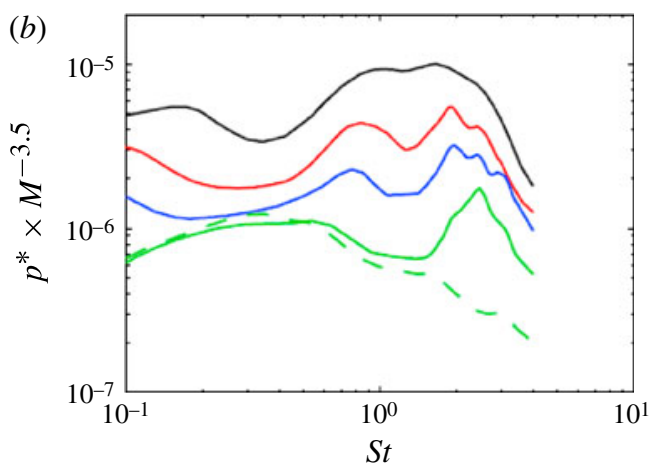

$-0.7 \%$

$0.5 \%$

FIGURE 1. Measured far-field sound spectra for laminar jets of different boundary layer nozzle thicknesses at radiated angles to the jet of $(a) 30^{\circ}$ and $(b) 90^{\circ}$ (Zaman 1985a); $p^{*}$ is power spectra density $\left[\mathrm{Pa}^{2} / \mathrm{St}\right]$ and $\mathrm{M}$ is jet Mach number measured at the nozzle exit. The plots are based on digitized data from the original publication of Zaman (1985a).

It should be noted that the laminar jet cases under consideration have qualitatively different noise spectra when compared with typical fully turbulent jets. The latter exhibit a considerably narrower $30^{\circ}$ spectrum in comparison with the $90^{\circ}$ one and a broad shape that peaks at around $S t=0.2$. The differences are due to the vortex roll-up and pairing that occur in the thin early shear layers of the initially laminar jets leading to an additional high-frequency peak in the spectra that is superimposed on the broad shape of the mixing noise.

Figure 1 shows the experimental noise spectra of several initially laminar jets from Zaman (1985a). The results for the jet of the smallest initial boundary thickness $(0.5 \%)$ without tripping are shown with a green solid line, and those for the tripped jet of the same thickness are shown with a dashed line. It can be observed that the peak noise frequency of the tripped jet and the peak frequency corresponding to the broad hump in the spectra of the untripped jet are about $S t \sim 0.2$ which is typical of the broad mixing noise of high-Reynolds-number jets.

In the numerical simulation of the initially laminar jets by Bogey \& Bailly (2010), the vortex pairing event occurs at the lip-line location $\left(r=r_{0}=D / 2\right)$ at around $z=0.45 D$ for the tripped jet and at $z=0.8 D$ for the untripped one. The LES calculation domain used by Bogey \& Bailly is terminated at the downstream end by a sponge zone starting at approximately 12.6 jet diameters from the nozzle exit. Their acoustic predictions were obtained by matching the LES solutions to an isotropic linearized Euler equations (ILEE) model on an open control surface, which covered the full axial domain, at a radial distance of 2.6 jet diameters from the jet axis.

In the present paper, a method based on the generalized acoustic analogy of Goldstein $(2002,2003)$ is used based on statistics reprocessed from unsteady solution data stored at the time of the initial Bogey \& Bailly calculations. The aims of the original paper were to study the initial shear layer development and subsequent evolution of the early part of the jet. Because of this, the unsteady solution stored was tailored to capture the potential core region of the jet and has some limitations for the purposes of this paper (to be discussed in $\S 4$ ). The two sets of LES data for 


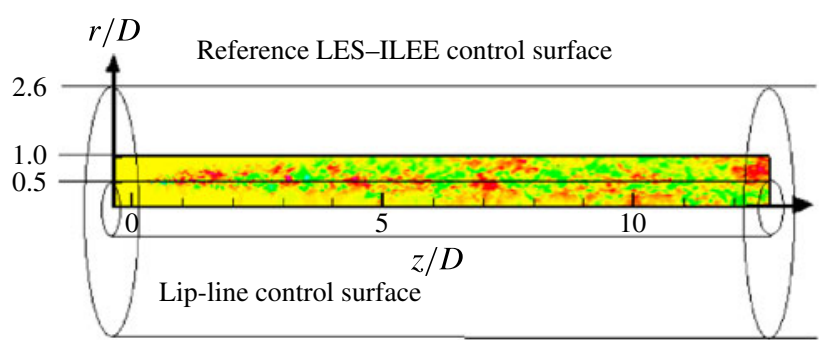

FIGURE 2. Input LES data definition delineating the area available for the acoustic analogy source estimation; the control surface location of the reference LES-ILEE is also shown.

the tripped and untripped jet are available only in one jet symmetry plane and at one cylindrical surface (at the nozzle lip-line location). The grid density corresponds to the original $595 \times 256 \times 249$ LES calculation. The spatial spread of the data available in the symmetry plane is 12.5 jet diameters axially and 1 jet diameter radially. The data sampling rate is $\Delta t=0.05 D / U$ and 4307 time samples in total are available for acoustic post-processing. A schematic of the computational setup of the problem is shown in figure 2. Because of the limited LES fields in the circumferential direction, most of the acoustic analogy modelling will be restricted to analysis using the in-plane data. The correlation length scale for variations in the circumferential and radial directions will, at each position in the jet, be assumed to be the same as that derived for variations in the axial direction, as in Karabasov et al. (2010). The study of the effect of correlation length scale anisotropy on noise will be a subject of future work.

Figure 3 shows typical instantaneous snapshots of axial velocity fluctuations computed from the LES-data fields in the symmetry plane of the two jets. The velocity fluctuations are defined with respect to the local time-mean density-weighted velocity field. To aid visibility, an axis ratio 1:10 (axial versus radial) is used here and throughout the rest of the paper. The role of velocity fluctuations in jet noise modelling will be discussed in $\S 3$ and some preliminary qualitative considerations are given below.

Figure 3(a-d) shows several consecutive snapshots of the velocity fluctuation field for the jet with no forcing. The velocity fluctuations show a seemingly repetitive cycle of complex dynamics in which the 'bursts' of large-scale structures appear in the early shear layers (figure $3 b$ ) then grow (figure $3 c$ ) and convect further downstream (figure $3 d, a$ ) as the jet broadens.

The velocity fluctuations of both jets, with and without forcing, show a qualitatively similar behaviour. For example, figures $3(e)$ and $3(f)$ compare typical instantaneous velocity fluctuation snapshots of the two jets. Black lines correspond approximately to the loci of $99 \%$ and $1 \%$ of mean axial velocity that mark the location of the mixing layer edges. It can be seen that the velocity fluctuation fields of the two different jets look similar for the first 10 diameters. If there were not a slight difference in the end of the jet potential core location, these flow snapshots could even be regarded as different realizations of the same turbulent flow.

\section{Acoustic analogy modelling}

The acoustic analogy used in this study follows the formulation described in Karabasov et al. (2010). The following system of LEE in the Goldstein (2002) 
(a)

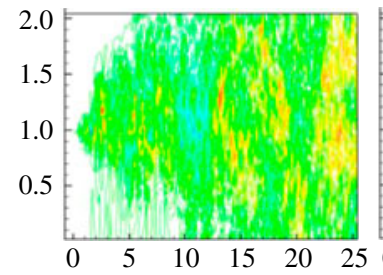

(e)
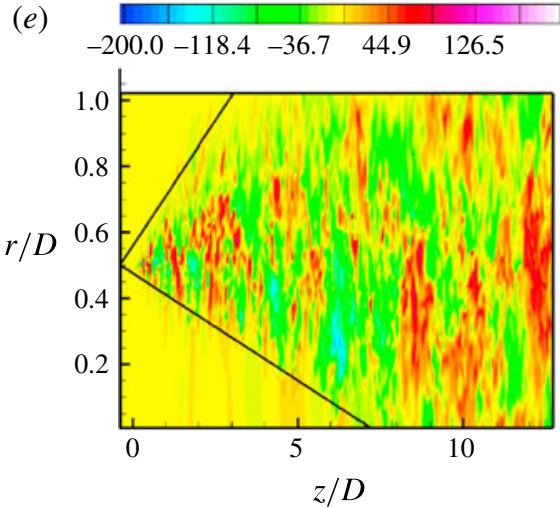

(c)

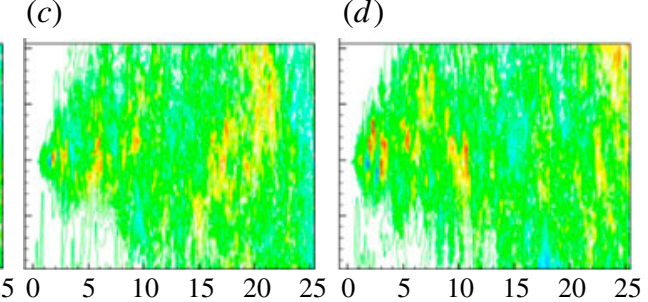

$(d)$ (b)

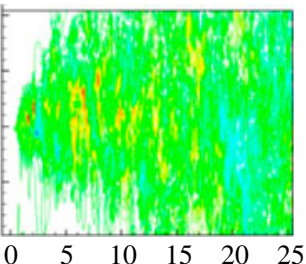

FIGURE 3. Instantaneous snapshots of velocity fluctuations in an untripped jet for consecutive time moments $(a-d)$ and zoomed-in fluctuations in $(e)$ a tripped jet and $(f)$ an untripped jet.

formulation in the source (jet) coordinates $(\boldsymbol{y}, \tau)$ is used:

$$
\left.\begin{array}{l}
\frac{\partial \rho^{\prime}}{\partial \tau}+\frac{\partial}{\partial y_{j}}\left(\rho^{\prime} \tilde{v}_{j}+u_{j}\right)=0, \\
\frac{\partial u_{i}}{\partial \tau}+\frac{\partial}{\partial y_{j}}\left(\tilde{v}_{j} u_{i}\right)+\frac{\partial p^{\prime}}{\partial y_{i}}+u_{j} \frac{\partial \tilde{v}_{i}}{\partial y_{j}}-\left(\frac{\rho^{\prime}}{\bar{\rho}}\right) \frac{\partial \tilde{\tau}_{i j}}{\partial y_{j}}=\frac{\partial T_{i j}^{\prime}}{\partial y_{j}}, \quad i=1, \ldots, 3, \\
\left(\frac{1}{\gamma-1}\right) \frac{\partial p^{\prime}}{\partial \tau}+\left(\frac{1}{\gamma-1}\right) \frac{\partial}{\partial y_{j}}\left(p^{\prime} \tilde{v}_{j}\right)+\frac{\partial}{\partial y_{j}}\left(u_{j} \tilde{h}\right)+p^{\prime} \frac{\partial \tilde{v}_{j}}{\partial y_{j}}-\left(\frac{u_{i}}{\bar{\rho}}\right) \frac{\partial \tilde{\tau}_{i j}}{\partial y_{j}}=0,
\end{array}\right\}
$$

where $u_{i}=\rho v_{i}^{\prime \prime}$ and the fluctuations are defined with respect to the time-averaged (overbar) and Favre-averged, $\tilde{f}=\overline{\rho f} / \bar{\rho}$, values of density, pressure, velocity and enthalpy, respectively:

$$
\rho=\bar{\rho}+\rho^{\prime}, \quad p=\bar{p}+p^{\prime}, \quad v_{i}=\tilde{v}_{1}+v_{i}^{\prime \prime} \quad h=\tilde{h}+h^{\prime \prime} .
$$

In the above, the following definitions of the Favre-averaged viscous stress gradient and fluctuating Reynolds stress are used:

$$
\frac{\partial \tilde{\tau}_{i j}}{\partial y_{j}}=-\frac{\partial}{\partial y_{j}}\left(\bar{\rho} \tilde{v}_{i} \tilde{v}_{j}\right) \quad \text { and } \quad T_{i j}^{\prime}=-\left(\rho v_{i}^{\prime \prime} v_{j}^{\prime \prime}-\overline{\rho v_{i}^{\prime \prime} v_{j}^{\prime \prime}}\right) .
$$

It should be noted that the time-average of the fluctuating Reynolds stress tensor $T_{i j}^{\prime}$ in (3.2b) is identically zero, unlike the Lighthill stress tensor $T_{i j}$ in (1.1).

Equations (3.1) are identical to equations (14), (19) and (20) of Goldstein (2002) with the source term on the right-hand side of the energy equation (20) set equal to 
zero, which is expected to be a reasonably good approximation for isothermal jets at subsonic Mach numbers, so that only the fluctuating Reynolds stress source survives. The far-field sound predicted using this model is given by a convolution integral of the adjoint LEE Green's function tensor $\hat{I}_{i j}$ with the tensor of the fourth-order two-space two-time velocity correlation function

$$
\hat{P}(\boldsymbol{x}, \omega)=\int_{V_{\infty}(\boldsymbol{y})} \int_{\Delta} \hat{R}_{i j k l}(\boldsymbol{y}, \boldsymbol{\Delta}, \omega) \hat{I}_{i j}(\boldsymbol{y}, \omega \mid \boldsymbol{x}) \hat{I}_{i j}(\boldsymbol{y}+\boldsymbol{\Delta},-\omega \mid \boldsymbol{x}) \mathrm{d}^{3} \boldsymbol{\Delta} \mathrm{d}^{3} \boldsymbol{y},
$$

where

$$
\begin{aligned}
\hat{I}_{i j}(\boldsymbol{y}, \omega \mid \boldsymbol{x})= & \frac{\partial \hat{G}_{j}}{\partial y_{i}}(\boldsymbol{y}, \omega \mid \boldsymbol{x})-\left[\frac{\partial \tilde{v}_{j}}{\partial y_{i}}(\boldsymbol{y}) \hat{G}_{4}(\boldsymbol{y}, \omega \mid \boldsymbol{x})+\tilde{v}_{j}(\boldsymbol{y}) \frac{\partial \hat{G}_{4}}{\partial y_{i}}(\boldsymbol{y}, \omega \mid \boldsymbol{x})\right] \\
& +\frac{\delta_{i j}}{2}\left(\mathrm{i} \omega+\tilde{v}_{k} \frac{\partial}{\partial y_{k}}\right) \hat{G}_{4}(\boldsymbol{y}, \omega \mid \boldsymbol{x}),
\end{aligned}
$$

where $\omega$ is radial frequency and $\delta$ is an increment of the source coordinate.

In the above $\hat{G}_{0}, \hat{G}_{1}-\hat{G}_{3}$ and $\hat{G}_{4}$ are the five components of the adjoint vector Green's function at the observer (far-field microphone) location $\boldsymbol{x}$ which correspond to the density, three Cartesian velocity components, and pressure fluctuation of the adjoint LEE operator in the frequency domain, respectively:

$$
\left.\begin{array}{l}
\mathrm{i} \omega \hat{G}_{0}+\tilde{v}_{j} \frac{\partial \hat{G}_{o}}{\partial y_{j}}+\left(\frac{\hat{G}_{i}}{\bar{\rho}}\right) \frac{\partial \tilde{\tau}_{i j}}{\partial y_{j}}=0, \\
\mathrm{i} \omega \hat{G}_{j}+\frac{\partial \hat{G}_{0}}{\partial y_{j}}+\tilde{v}_{i} \frac{\partial \hat{G}_{j}}{\partial y_{i}}-\hat{G}_{i} \frac{\partial \tilde{v}_{i}}{\partial y_{j}}+\tilde{h} \frac{\partial \hat{G}_{4}}{\partial y_{j}}+\left(\frac{\hat{G}_{4}}{\bar{\rho}}\right) \frac{\partial \tilde{\tau}_{i j}}{\partial y_{i}}=0, \quad j=\overline{1 . .3,} \\
\left(\frac{\mathrm{i} \omega}{\gamma-1}\right) \hat{G}_{4}+\left(\frac{\tilde{v}_{j}}{\gamma-1}\right) \frac{\partial \hat{G}_{4}}{\partial y_{j}}-\hat{G}_{4} \frac{\partial \tilde{v}_{j}}{\partial y_{j}}+\frac{\partial \hat{G}_{j}}{\partial y_{j}}=\delta(\boldsymbol{y}-\boldsymbol{x}),
\end{array}\right\}
$$

where $\boldsymbol{y}$ is the source coordinate and $\boldsymbol{x}$ is the observer coordinate.

The fourth-order velocity correlation functions $\hat{R}_{i j k l}(\boldsymbol{y}, \boldsymbol{\Delta}, \omega)$, representing a Reynolds stress covariance, are obtained by time-averaging the unsteady LES solutions, where Favre-averages for the fluctuating quantities are used. The previous study of Karabasov et al. (2010) showed that the corresponding fourth-order correlation functions $\hat{R}_{i j k l}(\boldsymbol{y}, \boldsymbol{\Delta}, \omega)=\int R_{i j k l}(\boldsymbol{y}, \boldsymbol{\Delta}, \tau) \mathrm{e}^{-\mathrm{i} \omega \tau} \mathrm{d} \tau=\int \overline{T_{i j}^{\prime}(\boldsymbol{y}, t) T_{k l}^{\prime}(\boldsymbol{y}+\boldsymbol{\Delta}, t+\tau)} \mathrm{e}^{-\mathrm{i} \omega \tau} \mathrm{d} \tau$ in a number of acoustically important jet regions (e.g in the developed shear layer region at the axial position of the end of potential core) can be reasonably well approximated by an analytical Gaussian function characterized by amplitude, length and time scales

$$
\begin{aligned}
R_{i j k l}(\boldsymbol{y}, \boldsymbol{\Delta}, \tau)= & A_{i j k l}(\boldsymbol{y}) \exp \left[-\Delta_{1} /\left(\tilde{v}_{1} \cdot \tau_{s}(\boldsymbol{y})\right)-\ln n 2\left(\left(\Delta_{1}-\tilde{v}_{1} \cdot \tau\right)^{2} / l_{s z}^{2}(\boldsymbol{y})\right.\right. \\
& \left.\left.+\Delta_{2}^{2} / l_{s \theta}^{2}(\boldsymbol{y})+\Delta_{3}^{2} / l_{s r}^{2}(\boldsymbol{y})\right)\right] .
\end{aligned}
$$

It is useful to give a physical interpretation of the Gaussian model of the Reynolds stress variance in (3.5), which also implicitly defines the acoustic source characteristics such as correlation time and space scales, $l_{s z}, l_{s r}, l_{s \theta}, \tau_{s}$. The Gaussian model describes a temporal correlation of turbulence moving with a gross turbulence convection speed $\tilde{v}_{1}$, as directly defined from the correlation fit (and which should not be confused with the local jet mean flow velocity) and exponentially decaying with a spatial separation $\Delta$. For constant source parameters with a free-space Green's function, (3.3a) can be 
integrated analytically to relate the space-time scales to the sound integral through the transformed space-time correlation (for details, see Morris \& Farassat 2002 for instance).

An advantage of the Gaussian-fit model is its simplicity which helps in reducing the complexity of the multi-dimensional integral in (3.3a). On the other hand, the simple Gaussian model does not include several experimentally observed features such as oscillations at large time delays, $\tau$, that can be up to $10 \%$ of the peak correlation values (e.g. Karabasov et al. 2010). The Gaussian model also does not capture the cusp-like peaks at vanishingly small time delays $\tau \rightarrow 0$ and zero spatial separation, $\Delta=0$, which may affect the representation at very high frequencies. Incorporation of those extra features into the computational model will also be a subject of future work.

In the present work, the Gaussian-fit scales are computed from the LES data at every point of the jet symmetry plane where data are available, which is the region sketched in figure 2. In contrast to the original paper by Karabasov et al. (2010), the present work does not use a RANS interpolation step, which involves evaluating the length scales at selected points in the jet and then assuming that they vary in the same way as length scales and time scales as predicted by a RANS method.

The LEE are solved numerically using an efficient adjoint method in the frequency domain (Karabasov \& Hynes 2006). The adjoint method is based on the reciprocity relations between the corresponding direct and adjoint vector Green's function. Tam \& Auriault (1998) show that, for far-field sound predictions from the distributed sources in a jet at a relatively few microphone locations in the far field, the use of an adjoint vector Green's function technique leads to a significant reduction in the computational cost of solution for the LEE when compared to a direct approach.

In the current work, the method of computing the adjoint Green's function has been slightly modified compared with Karabasov \& Hynes (2006) and Karabasov et al. (2010). As before, the governing system of adjoint LEE is solved in the frequency domain in the cylindrical-polar coordinate system $\left(y_{1}, y_{2}, y_{3}\right)=(x, r, \theta),\left(x_{1}, x_{2}, x_{3}\right)=$ $\left(x_{o}, r_{o}, \theta_{o}\right)$, where subscript ' $o$ ' indicates the observer. Because of the mean flow axisymmetry, the three-dimensional adjoint LEEs decouple into separate equations for each azimuthal mode $n: \boldsymbol{L}^{*} \boldsymbol{G}_{n}=0$, where

$$
\boldsymbol{L}^{*} \boldsymbol{G}_{n}=\left(\begin{array}{rr}
\mathrm{i} \omega \rho_{n}+\bar{u} \frac{\partial}{\partial x} \rho_{n} & +\bar{v} \frac{\partial}{\partial r} \rho_{n}+\frac{1}{\bar{\rho}}\left(u_{n} \frac{\partial}{\partial y_{\alpha}} \bar{\tau}_{x y_{\alpha}}+v_{n} \frac{\partial}{\partial y_{\alpha}} \bar{\tau}_{r y_{\alpha}}\right) \\
\mathrm{i} \omega u_{n}+\bar{u} \frac{\partial}{\partial x} u_{n}+\gamma \frac{\bar{p}}{\bar{\rho}} \frac{\partial}{\partial x} p_{n} & +\bar{v} \frac{\partial}{\partial r} u_{n}+\frac{\partial}{\partial x} \rho_{n}-u_{n} \frac{\partial}{\partial x} \bar{u}-v_{n} \frac{\partial}{\partial x} \bar{v} \\
i \omega v_{n}+\bar{u} \frac{\partial}{\partial x} v_{n}+\gamma \frac{\bar{p}}{\bar{\rho}} \frac{\partial}{\partial r} p_{n}-u_{n} \frac{\partial}{\partial r} \bar{u} & +(\gamma-1) \frac{p_{n}}{\bar{\rho}} \frac{\partial}{\partial y_{\alpha}} \bar{\tau}_{x y_{\alpha}} \\
i \omega w_{n}+\bar{u} \frac{\partial}{\partial x} w_{n}-\gamma \frac{\bar{p}}{\bar{\rho}} \frac{n}{r} p_{n} & +\bar{v} \frac{\partial}{\partial r} v_{n}+\frac{\partial}{\partial r} \rho_{n}-v_{n} \frac{\partial}{\partial r} \bar{v} \\
i \omega p_{n}+\bar{u} \frac{\partial}{\partial x} p_{n}+\frac{\partial}{\partial x} u_{n}+\frac{1}{r} \frac{\partial}{\partial r}\left(r v_{n}\right) & +(\gamma-1) \frac{p_{n}}{\bar{\rho}} \frac{\partial}{\partial y_{\alpha}} \bar{\tau}_{r y_{\alpha}} \\
& +\bar{v} \frac{\partial}{\partial r} w_{n}-\frac{n}{r} \rho_{n}-\frac{\bar{v}}{r} w_{n} \\
& +\bar{v} \frac{\partial}{\partial r} p_{n}+\frac{n}{r} w_{n}-(\gamma-1) p_{n} \\
& \times\left(\frac{\partial}{\partial x} \bar{u}+\frac{1}{r} \frac{\partial}{\partial r}(r \bar{v})\right)
\end{array}\right),
$$


which are solved with respect to the scattered component of the Green's function $\boldsymbol{G}^{s}=$ $\boldsymbol{G}-\boldsymbol{G}^{\text {incident }}$. In the above, $\boldsymbol{G}^{\text {incident }}$, which for the simplest case of $\boldsymbol{G}^{\text {incident }}=\boldsymbol{G}^{\text {free-space }}$ is determined analytically, is the incident wave solution that satisfies to the corresponding reciprocal boundary conditions which correspond to a point sink at the observer location (the delta-function in the last equation of (3.4)). The full solution is obtained through the contribution of all azimuthal modes and five individual components

$$
\boldsymbol{G}^{s}=\sum_{n=0}^{\infty} \boldsymbol{G}_{n}^{s}=\sum_{n=0}^{\infty}\left(\rho_{n}^{s} \cos \theta^{\prime}, u_{n}^{s} \cos \theta^{\prime}, v_{n}^{s} \cos \theta^{\prime}, w_{n}^{s} \sin \theta^{\prime}, p_{n}^{s}(\gamma-1) \cos \theta^{\prime}\right)^{\mathrm{T}},
$$

where $\theta^{\prime}=n\left(\theta-\theta_{o}\right)$ is $n$ times the circumferential angle in the jet with respect to the observer location, and $\rho_{n}^{s},\left(u_{n}^{s}, v_{n}^{s}, w_{n}^{s}\right), p_{n}^{s}$ are the adjoint density, velocity and pressure variables per mode in a cylindrical-polar coordinate system.

The resulting elliptic problem for each mode is discretized with second-order central differences for the partial derivatives and solved iteratively with a time-like method based on a modified Adams scheme with carefully selected time steps to suppress any numerical instability (Karabasov \& Hynes 2006). In contrast to that previous work, the domain for the propagation calculation used in the current study does not include the nozzle lip. Instead, the computational domain for the propagation calculation is extended upstream of the nozzle exit as a parallel jet flow with the flow profile at the jet nozzle exit, and non-reflecting boundary conditions of characteristic-type with a sponge buffer layer are used at all open boundaries. A similar technique is used for the open boundary at the jet outflow where the corresponding outflow profile is extended as a semi-analytic parallel flow solution matched to the numerical LEE solution. This extension is implemented as a non-reflecting sponge zone that spans several jet diameters. A further slight variation, that improves the conditioning of the calculation procedure, is that the solution is found as a scattered wave component, by letting $\boldsymbol{G}^{\text {incident }}=\boldsymbol{G}^{\text {locally parallel }}$ and $\boldsymbol{G}^{s}=\boldsymbol{G}-\boldsymbol{G}^{\text {locally parallel }}$, relative to the Green's function that corresponds to the solution for locally parallel jet wave scattering. The terms which correspond to the parallel mean flow effects are grouped on the left-hand side of the adjoint LEE propagator (3.6) and retaining only those terms reduces the original scattering problem to the solution of a straightforward set of ordinary differential equations. The method outlined in Karabasov \& Hynes (2006) uses a scattered component relative to a free-space solution, $\boldsymbol{G}^{\text {incident }}=\boldsymbol{G}^{\text {free-space }}$. The main advantage of choosing to find the solution as the difference from the locally parallel solution, $\boldsymbol{G}^{\text {incident }}=\boldsymbol{G}^{\text {locally parallel }}$ is that the sponge zones exhibit a numerically superior nonreflecting property when compared with those which sponge the numerical solution towards a target solution with no jet. The numerical methodology has been validated against simplified test problems such as plane acoustic wave scattering by a parallel jet flow (e.g. Karabasov \& Hynes 2006) and its detailed description will be the subject of a separate work. For the purpose of the current work, which concentrates on the physical mechanisms of sound generation, the numerical method of computing the adjoint LEE vector Green's function is validated as a part of the complete acoustic model, including the source, in comparison with the reference LES-ILEE solution of Bogey \& Bailly in $\S 5$.

\section{Acoustic post-processing of the LES data}

For each position in the jet for which LES data are available, the fourth-order velocity correlations are first computed: 


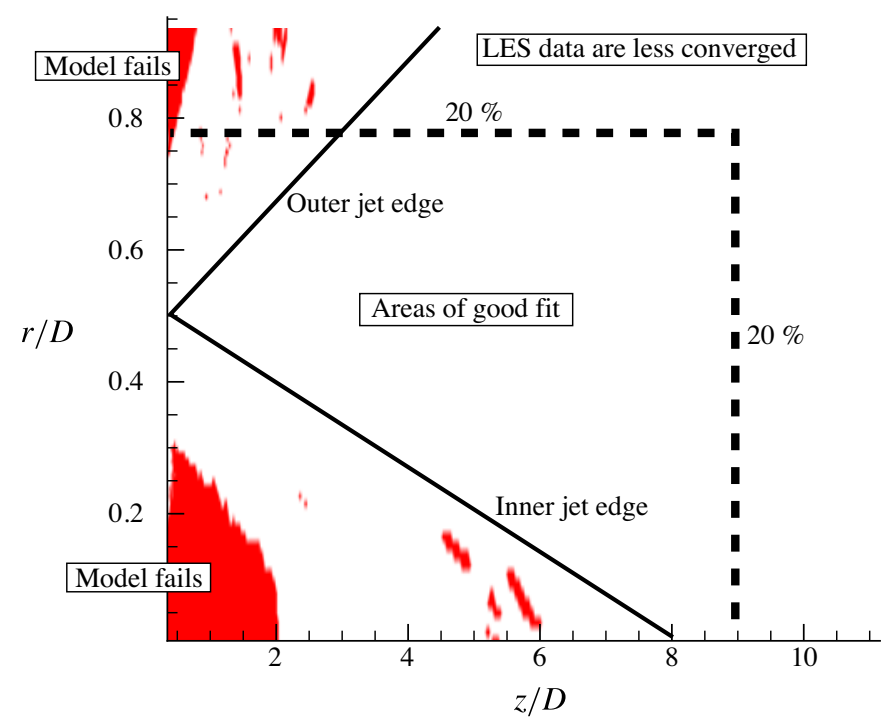

FIGURE 4. (Colour online) Schematic of the correlation fits for the tripped jet case. The less converged correlation data for large axial and radial locations corresponds to a $10-20 \%$ error for large space-time separations in the two-point two-time correction function.

$$
\begin{aligned}
& R_{i j k l}(\boldsymbol{y}, \mathrm{d} x, \mathrm{~d} t) \\
&= \overline{\rho(\boldsymbol{y}+\mathrm{d} x, \tau+\mathrm{d} t) v_{i}^{\prime \prime}(\boldsymbol{y}+\mathrm{d} x, \tau+\mathrm{d} t) v_{j}^{\prime \prime}(\boldsymbol{y}+\mathrm{d} x, \tau+\mathrm{d} t) \rho(\boldsymbol{y}, \tau) v_{k}^{\prime \prime}(\boldsymbol{y}, \tau) v_{l}^{\prime \prime}(\boldsymbol{y}, \tau)} \\
&-\overline{\rho(\boldsymbol{y}+\mathrm{d} x, \tau+\mathrm{d} t) v_{i}^{\prime \prime}(\boldsymbol{y}+\mathrm{d} x, \tau+\mathrm{d} t) v_{j}^{\prime \prime}(\boldsymbol{y}+\mathrm{d} x, \tau+\mathrm{d} t)} \cdot \overline{\rho(\boldsymbol{y}, \tau) v_{k}^{\prime \prime}(\boldsymbol{y}, \tau) v_{l}^{\prime \prime}(\boldsymbol{y}, \tau)}
\end{aligned}
$$

Here $i, j, k, l=1,2,3$ are local Cartesian directions aligned with the axial, circumferential and radial directions of the cylindrical-polar coordinate system respectively. The next step is to find a fit to these numerical data using the analytical Gaussian function (3.3a). Details of the numerical methodology of applying the Gaussian fit to the fourth-order correlations computed directly from the LES data samples are described in the Appendix. Here a summary of the results is provided.

Overall, the Gaussian-fit model provides a good approximation to the fourth-order statistics of the LES solution for most points in the jet except for some points close to the nozzle exit and also some jet locations at the jet edge, where the contribution to the acoustic integral is likely to be small because the amplitude of the corresponding correlation amplitudes is small. A more notable effect on the validity range of the acoustic source model is expected from outer regions of the jet that correspond to large axial $z \sim 10 D-12 D$ and radial $r \sim 0.8 D-1 D$ locations. For these locations, the LES data appear to be less well converged and show some 10-20\% errors for the two-point two-time correlation statistics at large space separations and time delays.

For future reference, it is useful to draw a schematic of the regions of the jet where the Gaussian correlation fit appears to work well, where the fits appear to be not well converged and where they fail completely. Figure 4 shows such a schematic for the tripped case (for the untripped jet the decomposition of the jet field is similar).

The above information can be used to assess the limits of applicability of the overall acoustic analogy model for the tripped jet case that bears some similarities with the solutions obtained for a turbulent jet exhaust aerodynamics and noise (JEAN) 

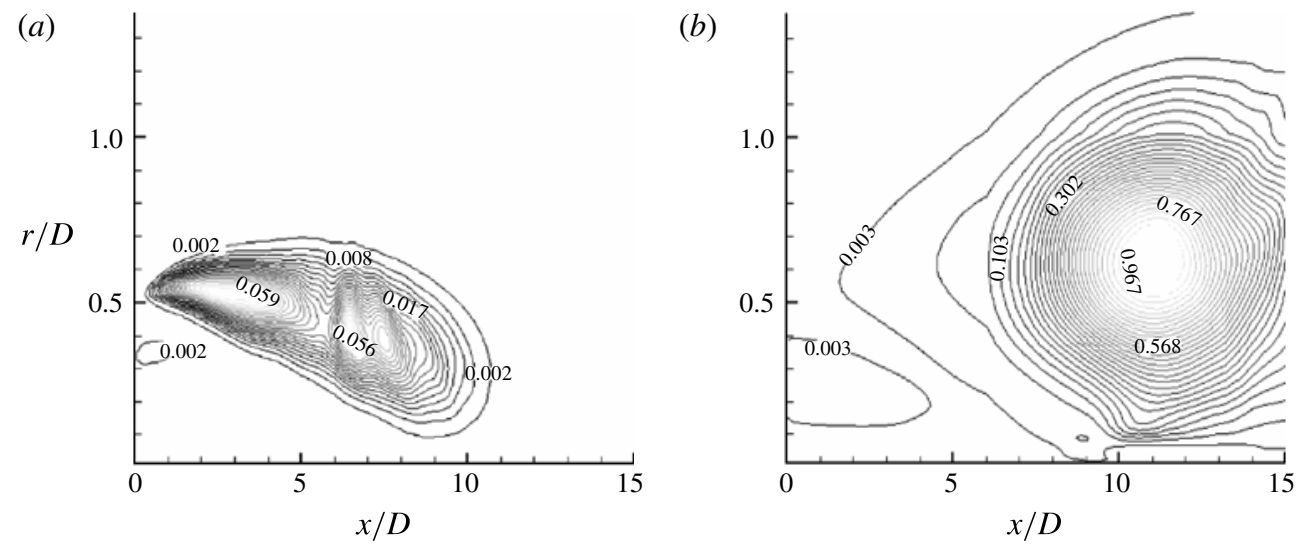

FIGURE 5. Efficient acoustic source distribution for the turbulent JEAN case at $30^{\circ}$ observer location from Karabasov et al. (2010) for: (a) $S t=1$; and (b) $S t=0.2$.
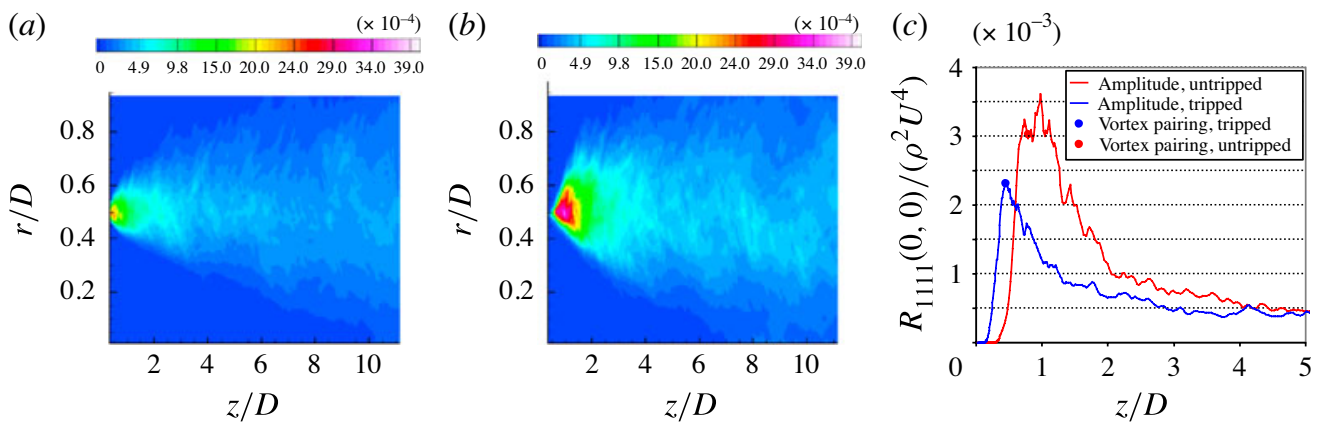

FIGURE 6. In-plane autocorrelation field of the longitudinal component $\left(R_{1111}\right)$ for $(a)$ a tripped jet and $(b)$ an untripped jet; and $(c)$ the lip-line distributions for the two jets, including vortex-pairing locations from Bogey \& Bailly (2010).

case (at higher $R e=10^{6}$ ) considered in Karabasov et al. (2010), and in Power et al. (2004). Figure 5 shows the efficiently radiating acoustic source distribution (i.e. when weighted with the propagation term $\hat{I}_{i j} \hat{I}_{k l}$ in $(3.3 a)$ ) of the JEAN model at $30^{\circ}$ relative to the downstream jet direction for $S t=0.2$ and $S t=1$. Given the present LES data limitations, which are effectively bracketed by $z \sim 9 D$ in the axial direction for low frequencies, it is reasonable to expect that the low-frequency limit of the applicability of the acoustic noise prediction for the current study lies between $0.2<S t<1$.

The main outcome of the Gaussian-fit procedure is the prediction of the characteristic amplitude, and space and time scales of the acoustic source for each jet location. Figure $6(a, b)$ shows the results for the autocorrelation scale distribution in the $z-r$ data plane for the tripped and untripped jets for the largest noise source component $R_{1111}$. Figure $6(c)$ shows the corresponding profile at the lip radius. Also marked are the locations of shear-layer vortex pairing events as found and discussed by Bogey \& Bailly (2010). 

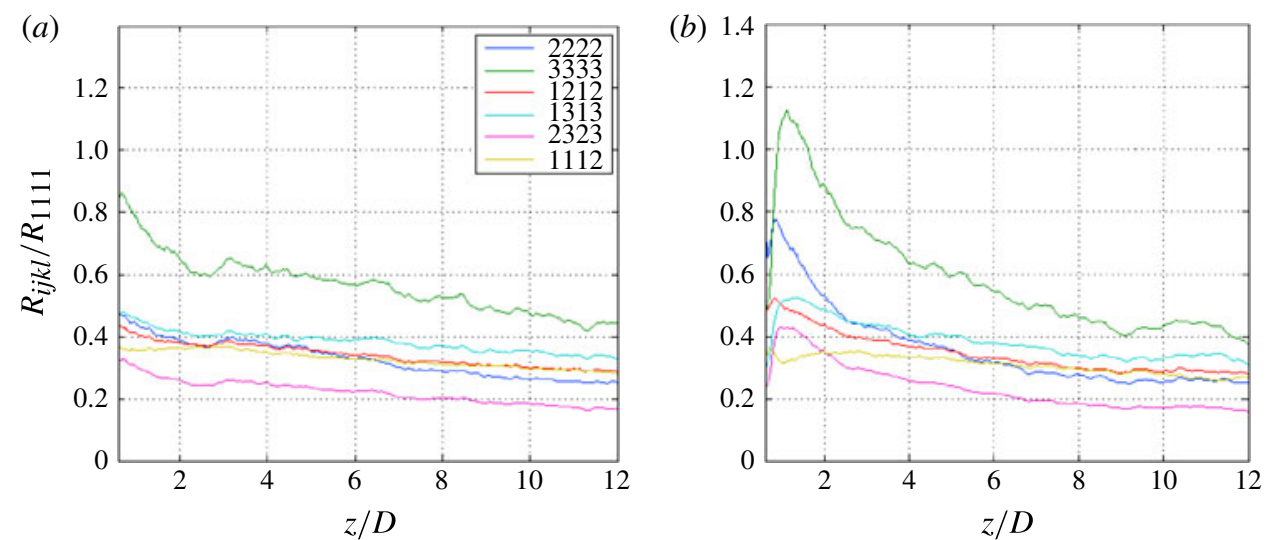

FIGURE 7. Relative amplitude ( $i j k l$ ) distribution for the most significant source components for $(a)$ a tripped and $(b)$ an untripped jet. Data averaging around the circumference is applied.

Figure 7 shows the lip-line distributions of autocorrelation amplitudes normalized by the longitudinal component $R_{1111}$. To improve convergence, circumferential averaging is applied (this radial location is the only one where data are available to do this). For the untripped jet, there is a notable amplification of the two correlation components that lie in the normal plane at the upstream end of the jet $\left(R_{2222}, R_{3333}\right)$, which may be associated with the initial development of the laminar shear layer. Apart from this effect, the relative amplitudes further downstream for the two jets are similar. In contrast to the fully turbulent jet case studied in Karabasov et al. (2010), here there is a relatively stronger contribution from the normal-plane correlation components $\left(R_{2222}, R_{3333}\right)$. The autocorrelations $\left(R_{1111}, R_{2222}, R_{3333}, R_{1212}, R_{1313}, R_{2323}\right)$ are precisely the same set of terms that were found to be significant by Karabasov et al. (2010). The one exception is a new non-small term associated with the axial-radial velocity interaction $\left(R_{1112}\right)$.

The correlation length scales in the axial, radial and circumferential directions along the lip line and for one $z-r$ plane slice of the data of the two jets are shown in figure 8. The uneven character of the spatial distribution of the correlation scales is associated with insufficient averaging time.

The correlation length scales correspond to a rate of change of the Gaussian correlation shapes (Appendix); hence, their spatial distribution is more sensitive to the numerical noise associated with the limited time-data series in comparison with the two-point two-time correlation curves that are smooth. Previous analysis of Karabasov et al. (2010) confirmed that the Goldstein acoustic analogy is relatively robust in terms of a moderate dependence of the far-field predictions on the uncertainty of the correlation scale determination $(10 \%$ variation in the length scales corresponds to approximately $1 \mathrm{~dB}$ variation in jet noise predictions).

There is a prominent amplification of the circumferential length scale at the upstream end for the case of the untripped jet, which may be associated with the laminar inflow boundary condition for this case. Apart from the peak at the beginning of the jet in the untripped case, the scales of both jets look similar. The relative scale values of the fourth-order correlations of these initially laminar jets (axial length scale to radial scale is approximately 3 to 1 and circumferential scale to radial scale is approximately 3 to 2 ) are in good agreement with the values found experimentally on 
(a)

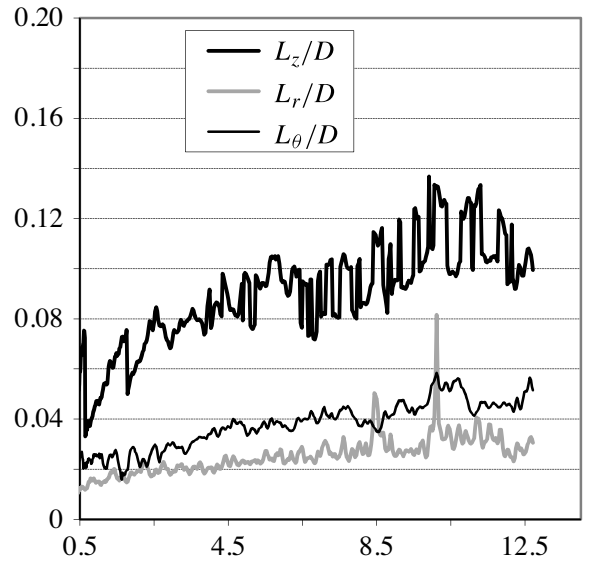

(c)

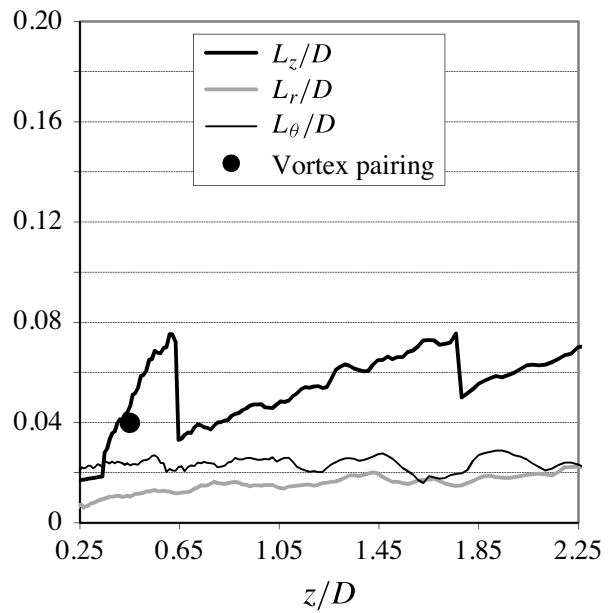

(b) 0.20

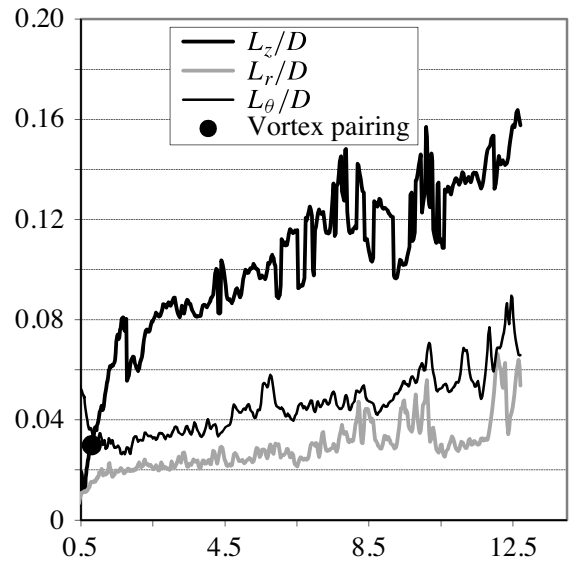

(d)

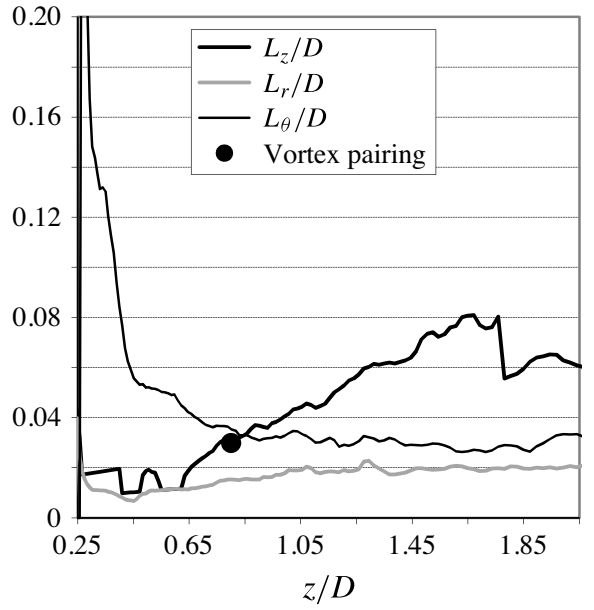

FIGURE 8. In-plane correlation length scales along the lip line for $(a, c)$ a tripped jet and $(b, d)$ an untripped jet; locations of vortex pairing from Bogey \& Bailly (2010) are also shown as a red dot.

the lip line location of a round jet experiment by Morris \& Zaman (2010). It is also interesting to note that the relative scales pertinent to the fourth-order correlations are somewhat different to those computed for the second-order correlations in Bogey et al. (2011a), where $L_{z} / L_{\theta}$, for example, is found to be $\sim 6$.

The spatial distribution of the axial correlation scales for both jets in the symmetry plane is shown in figure 9. The contours are chosen to highlight the correlation scales which contain most of the acoustic source. Specifically, the value of the maximum correlation length scale shown is $0.2 \mathrm{D}$, that corresponds to the maximum length scale of the effective acoustic source, detailed investigation of which will be the subject of $\S 6$. For now, there are several general observations that can be made. The correlation scales tend to grow with downstream and radial distance within the jets. There are regions of large scales emerging around the inner edge of both jets, which then decay with further downstream distance. The region of the large spatial scales is more prominent for the tripped jet, which has a larger potential core.

The distribution of the correlation time scale is obtained in a similar way. The time scales are similar for both jets, with the growth rate slightly larger for the untripped 
(a)
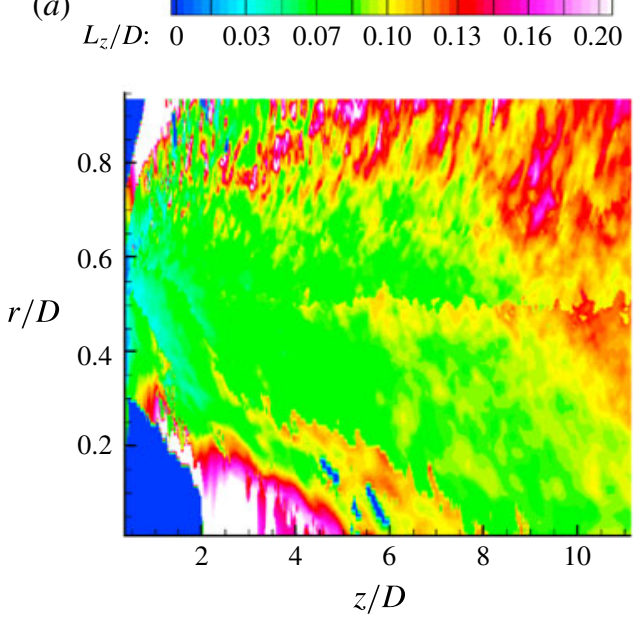

(b)
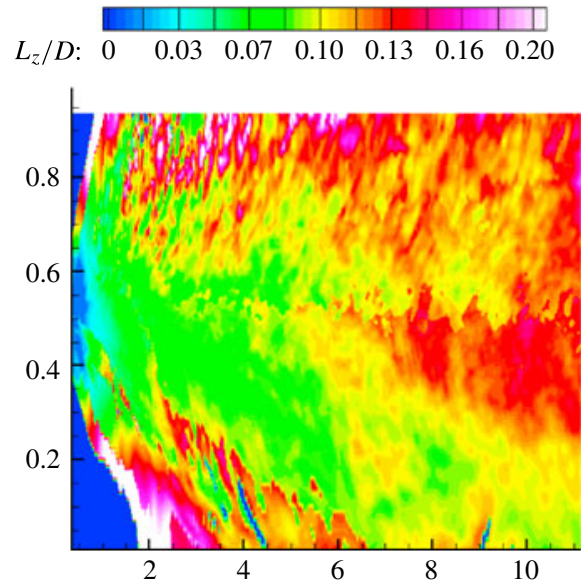

$z / D$

FIGURE 9. In-plane axial correlation length scales for $(a)$ a tripped and $(b)$ an untripped jet.
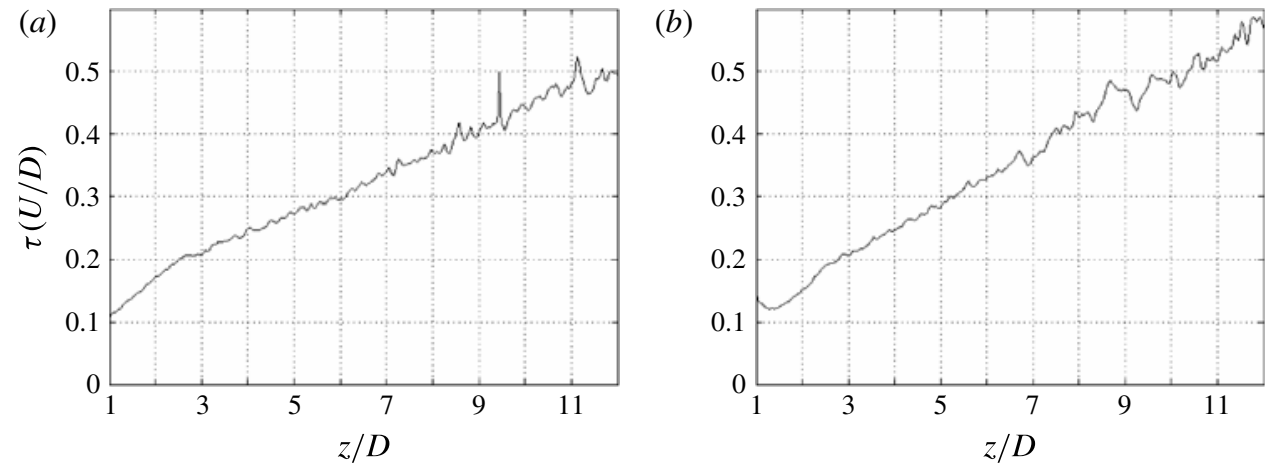

FIGURE 10. Temporal correlation length scales along the lip line for $(a)$ a tripped and $(b)$ an untripped jet. Data averaging around the circumference is applied.

jet, and they exhibit an approximately linear growth with axial distance starting from 3 jet diameters, similar to the observed variation of spatial correlation scales (cf. figure 10).

\section{Comparison of the far-field sound prediction results with the reference solution and assessment of the consistency of the model}

Figure 11 shows the predicted sound power spectral density (PSD) $[\mathrm{dB} / S t]$ for the two jets at $30^{\circ}$ and $90^{\circ}$ to the downstream axis for the frequency range focused on the high-frequency peak of the initially laminar jet noise spectra (cf. figure 1).

The observer location is taken to be 30 jet diameters from the nozzle exit. The acoustic analogy prediction is based on the solution of the linearized Euler equations (3.1) and the statistical source model that includes all the major terms, with the integral evaluated over the entire region for which there are LES data. The spectra 

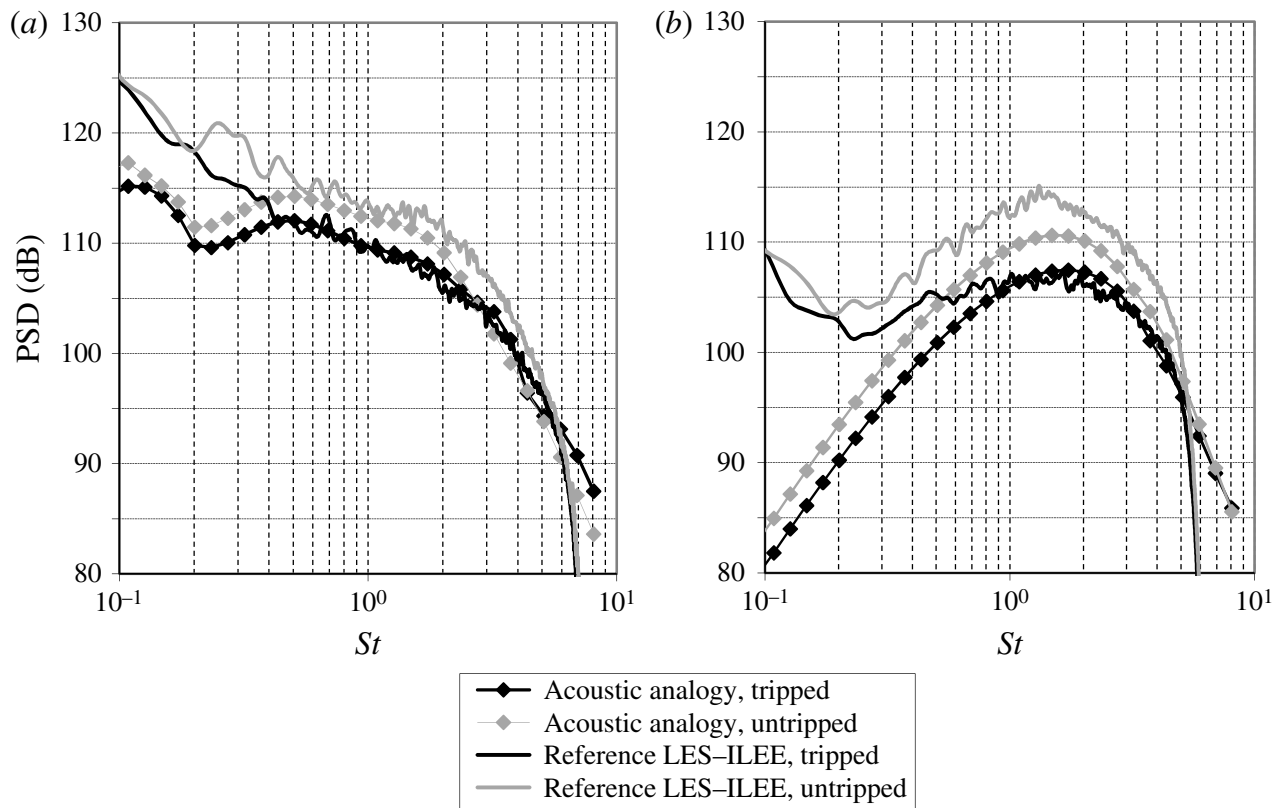

FIGURE 11. Predicted far-field spectra for $(a) 30^{\circ}$ and $(b) 90^{\circ}$ to the jet axis for the tripped jet and untripped jet: comparison with the reference LES-ILEE method.

of the reference solutions obtained with the open control surface LES-ILEE method from Bogey \& Bailly (2010) are shown in the same plots for comparison.

For the tripped case and for both angles to the jet the prediction of the acoustic analogy model is within $1 \mathrm{~dB}$ of the reference LES-ILEE solution for frequencies $0.8<S t<6$ (and within $2 \mathrm{~dB}$ for $0.5<S t<6$ ). For the untripped jet, the agreement between the two methods is less good and amounts to $3-4 \mathrm{~dB}$. The bigger discrepancy in comparison with the reference solution in the untripped jet case is associated with the very strong vortex-pairing that happens in this case, as identified by Bogey \& Bailly (2010), and which may be less well captured by the statistical acoustic source model in comparison with the tripped jet case. Also, the strong vortex pairing in the untripped case gives rise to an acoustic source that is dominated by longer spatial correlation scales that are distributed further downstream in the jet, which will be discussed in $\S 6$.

There are also some differences between the two predictions for both jets at high and low frequencies. The high-frequency discrepancy is caused by the grid cut-off frequency, above $S t=6$, imposed by the grid resolution at the LES-ILEE control surface location. The discrepancies for low frequencies, $S t<0.5$, between the two predictions are probably associated with insufficient time averaging and with the reduced extent of the spatial LES domain, as discussed in $\S 6$. For frequencies below $S t=0.1-0.2$, the reference LES-ILEE solution is also likely to be contaminated by numerical artifacts due to the open control surface, as acknowledged by Bogey \& Bailly (2010) in the Appendix of their paper.

The acoustic analogy model contains three key elements: (i) a statistical source model based on fourth-order velocity correlations that include all significant directivity components; (ii) mean flow sound propagation/interaction through the solution of 

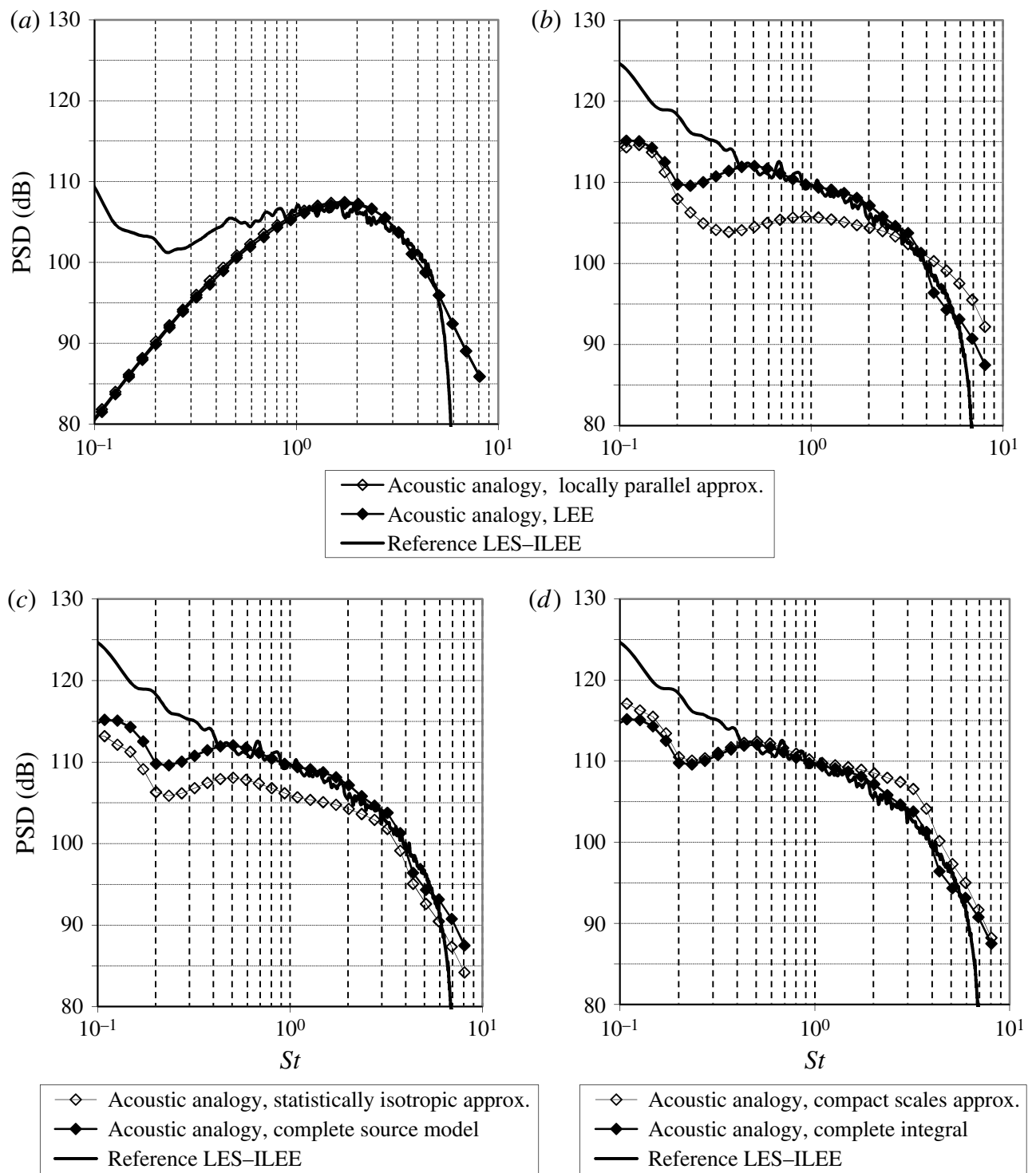

FIGURE 12. Effect of the locally parallel jet approximation at $(a) 90^{\circ}$ and $(b) 30^{\circ}$ to the tripped jet. $(c)$ Effect of assuming compact integral scales; and $(d)$ statistically isotropic source.

linearized Euler equations; and (iii) non-compact acoustic integration that includes the full source/Green's function convolution in space. For the fully turbulent JEAN jet, Karabasov et al. (2010) show that all three elements can be crucial for accurate sound predictions. The same study is repeated here for the tripped jet case.

Figure 12 $(a, b)$ compares the result of the locally parallel jet approximation with the full linearized Euler propagation model for different angles to the jet. While at $90^{\circ}$ the difference is insignificant, at $30^{\circ}$ the error due to neglecting the jet spreading can be as large as $5 \mathrm{~dB}$ for high frequencies and $8 \mathrm{~dB}$ for low frequencies. This result reinforces the conclusions of the work of Karabasov et al. (2010) and Karabasov 
(2010) who found that the explicit accounting for the mean flow-distributed sound source interaction effects in the framework of LEE can be very important for capturing the peak directivity noise.

Figure 12(c) compares the result of completing a full integration with a 'compact' approximation, i.e. neglecting the source variation in the radial direction in comparison with the propagation scale so that the full convolution reduces to a single-space integration. The error due to the compact scales approximation is not as marked as that due to using the locally parallel model. It is within $3-4 \mathrm{~dB}$ and for many frequencies is less than $2 \mathrm{~dB}$. For the same compact model, figure $12(d)$ compares the result of the full source model based on seven anisotropic components with the statistically isotropic model that assumes symmetry of the fourth-order correlation tensor components similar to the turbulent acoustic source model described in Afsar (2010). For the present tripped jet case, it appears that the isotropic approximation leads to a $5 \mathrm{~dB}$ error in sound pressure levels.

\section{Noise sources}

One of the main advantages of the acoustic analogy method is that it provides information about the location of efficiently radiating acoustic equivalent sources in the jet. The efficient noise source data include both the noise generation $R_{i j k l}$ obtained from LES and the propagation term $\hat{I}_{i j} \hat{I}_{k l}$ which accounts for the distributed source-mean flow interaction, and which can be very important at small angles to the jet, as discussed earlier. In the following figures, the spatial distribution of the integrand in (3.3a) weighted with radius is used as an effective noise source density. Its integral over the jet area in $(z, r)$ coordinates amounts to the power spectral density at the far-field observer location and the local maxima correspond to the peak noise sources within the jet.

Figure 13 shows the noise source density distribution within the tripped jet $(a, c)$ and untripped jet $(b, d)$ at $30^{\circ}$ and $90^{\circ}$ to the jet axis for the characteristic frequency $S t=1$. For each case, the field is normalized by the peak value. In order to estimate the relative source importance with position within the jet, the contribution to the full sound integral of the acoustic sources which are located in the furthest downstream locations, $z=10 D-12 D$, of the LES spatial domain in the axial direction (right-hand $20 \%$ of the domain), is computed. A similar exercise for the radial direction involves computing the contribution from the locations, $r=0.8 D-1 D$, in the radial direction (the top $20 \%$ of the domain). The results for each jet and $30^{\circ}$ and $90^{\circ}$ angles at $S t=1$ are presented in table 1 . This is to be compared with table 2 which shows results for the tripped case at $S t=0.3$.

As can be seen from the tables, as far as radial extent is concerned, the major contributions to noise for both the tripped and the untripped jets are within the region covered by the LES data, with the top $20 \%(0.2 D)$ of this domain contributing less than $5 \%$ of the acoustic energy. For the axial extent, the source fields of both jets are significantly distributed over a large portion of the jet domain. The relative weight of the last $20 \%$ of the area at the end of the potential core $(10 D-12 D)$ is larger for the small observer angle in comparison with $90^{\circ}$. Also, the effective acoustic source for the untripped jet has a smaller contribution from the source distributed over the last $20 \%$ of the axial extent of the jet in comparison with the tripped case.

A straightforward explanation for the latter differences between the two jets is that the tripped jet has a longer potential core in comparison to the untripped one, leading to the larger acoustic source size. However, there is also the possibility that the 
(a)
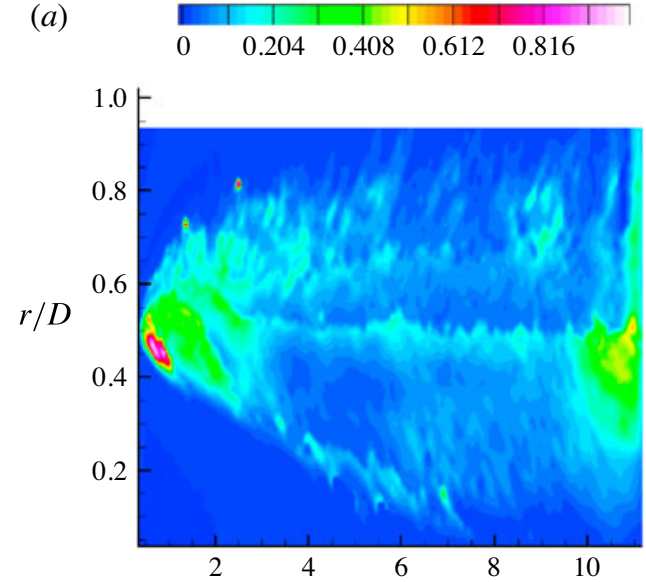

(c)

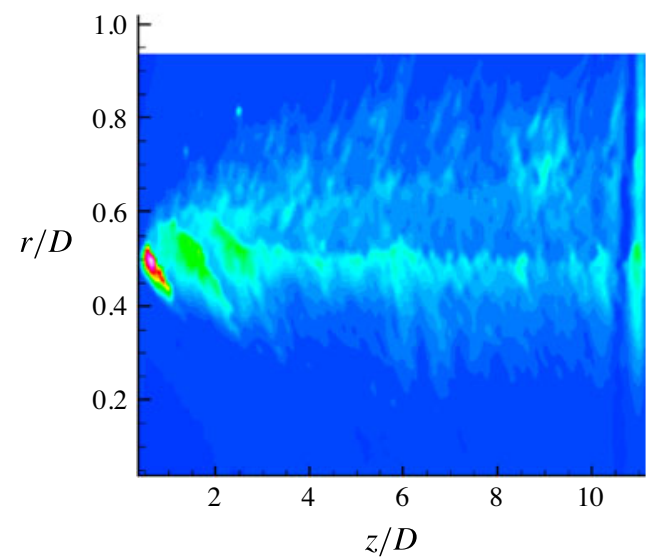

(b)
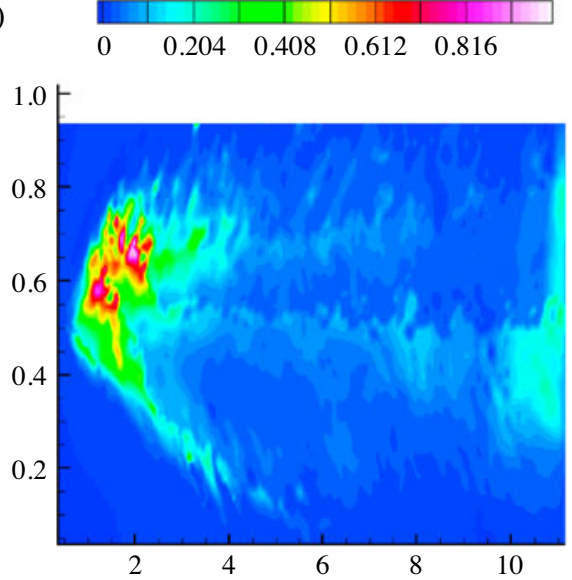

$(d)$
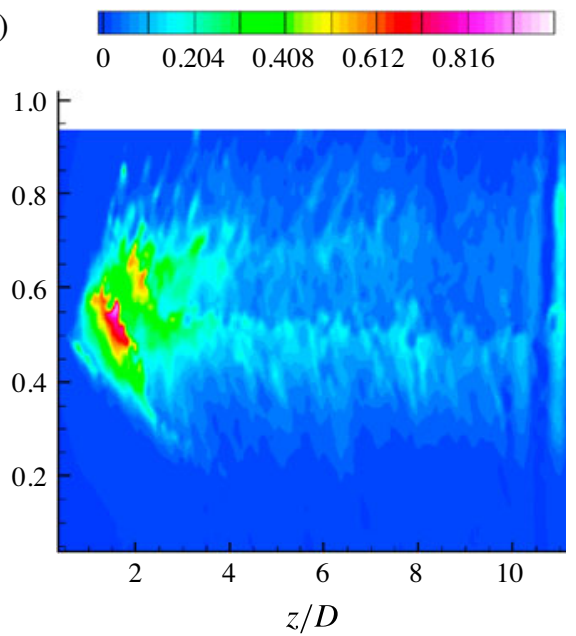

FIGURE 13. Acoustic source location at $S t=1$ for: $(a)$ tripped jet, observer at $30^{\circ}$; (b) untripped jet, observer $30^{\circ}$; (c) tripped jet, observer at $90^{\circ} ;(d)$ untripped jet, observer $90^{\circ}$.

important source regions are distributed over a greater axial extent for the untripped case in comparison with the tripped jet and, correspondingly, less of the total acoustic energy is contained within the first 10 jet diameters for the untripped case, meaning that the flow statistics are less well captured by the available LES data. It turns out that this may well be the case and this issue is discussed further in what is following.

Table 2 shows the results of the tripped jet at low frequency $(S t=0.3)$. The comparison of these results with the results of the same jet at high frequency (table 1) shows that, for the small angle to the jet, the contribution of the effective acoustic source at the downstream side of the jet $(z=10 D-12 D)$ is smaller by a factor of approximately 2 at the low frequency $(17 \%)$ in comparison with the high frequency $(30 \%)$. The apparent reduction of the axial acoustic source extent with frequency is non-physical because the acoustic source at low frequency is expected to be at least as non-compact as that at the high frequency (e.g. cf. figure 5). Hence, one can conclude that there is an important part of the low-frequency noise missing from the acoustic 


\begin{tabular}{lccc} 
& & $30^{\circ}$ angle $(\%)$ & $90^{\circ}$ angle $(\%)$ \\
Tripped jet & Right $2 D$ & 30 & 23 \\
\multirow{3}{*}{ Untripped jet } & Top 0.2D & 3 & 3 \\
& Right $2 D$ & 19 & 13 \\
& Top 0.2D & 4 & 5
\end{tabular}

TABLE 1. Relative contribution of the boundary areas to the acoustic integral at $S t=1$.

$\begin{array}{lcc} & 30^{\circ} \text { angle }(\%) & 90^{\circ} \text { angle }(\%) \\ \text { Right } 2 D & 17 & 29 \\ \text { Top0.2D } & 1 & 2\end{array}$

TABLE 2. Relative contribution of the boundary regions to the acoustic integral for the tripped case at $S t=0.3$.

(a)

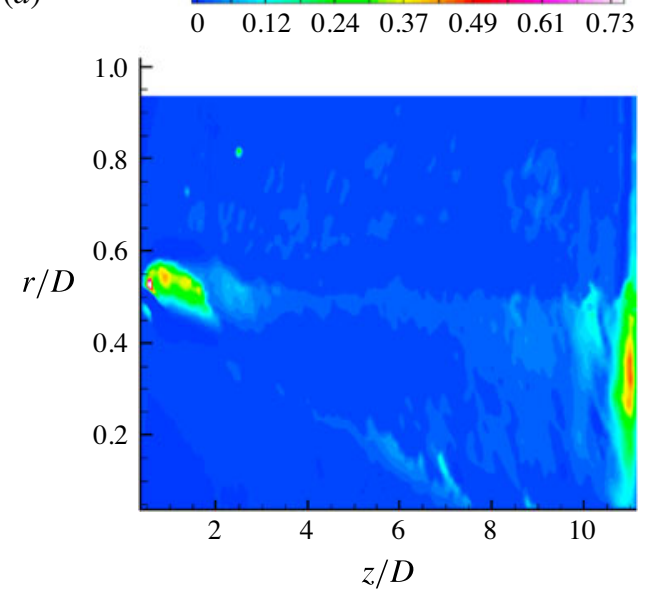

(b)

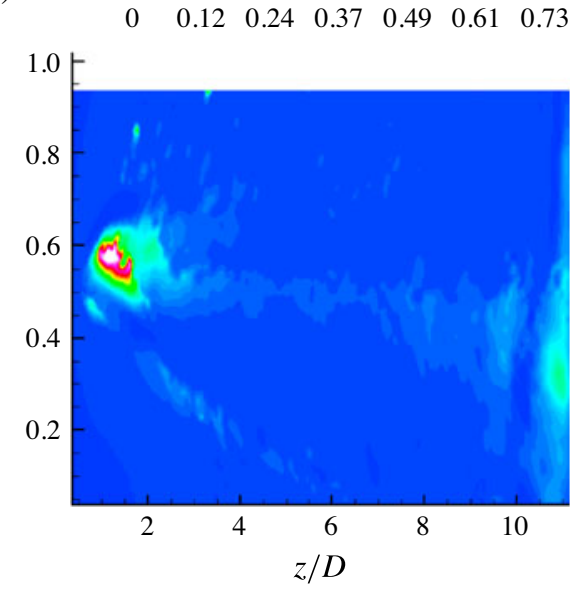

FIGURE 14. Acoustic sources at the vortex pairing frequency for the observer at $30^{\circ}$ to the jet axis for: $(a)$ tripped jet, $S t=2.16$; and $(b)$ untripped jet, $S t=1.61$.

model at the downstream area of the jet. This is most likely due to the lack of statistical convergence of LES data in this area.

Figure 14 shows the noise source densities of the two jets for the observer at $30^{\circ}$ to the jet axis, corresponding to $S t=2.16$ for the tripped jet case and $S t=1.61$ for the untripped one (the dominant frequencies for the vortex pairing source mechanism identified by Bogey \& Bailly). The biggest amplitudes of the sources are located at the upstream end of the jet close to the vortex pairing region and the peak source locations identified here are very similar to those reported in Bogey \& Bailly. For lip-line locations, these are $\sim 0.45 D$ for the tripped jet and $0.8 D$ for the untripped case.

A deeper insight into which scales are the dominant overall contributors to the radiated noise and which regions of the jet contribute them can be inferred from 
(a)

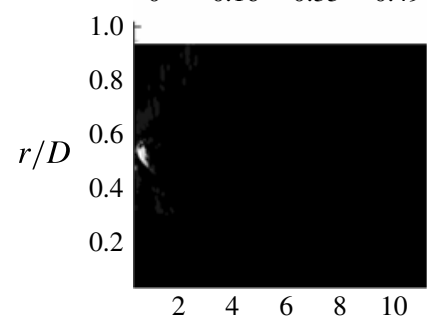

(d)

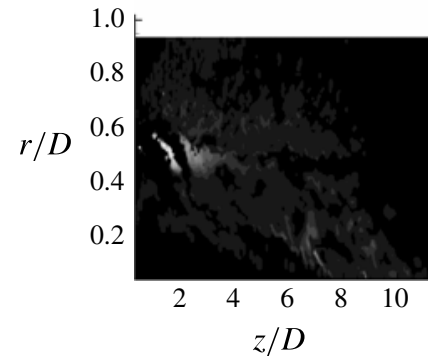

(b)

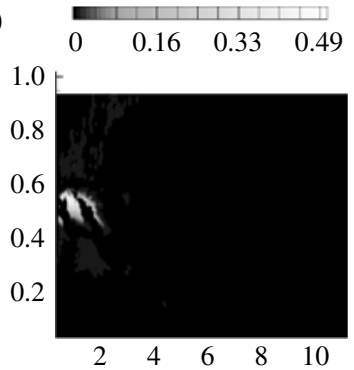

$(e)$

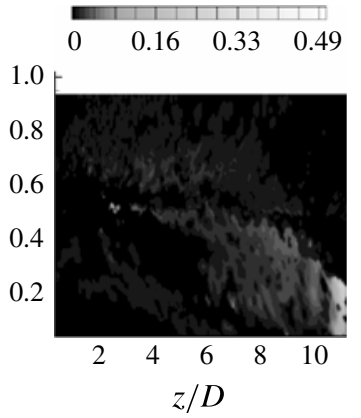

$(c)$

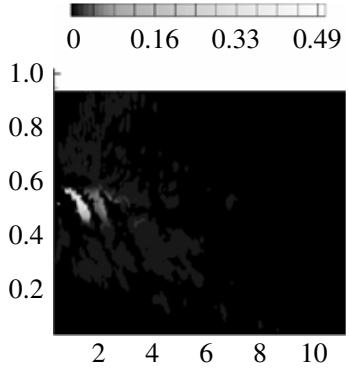

(f)

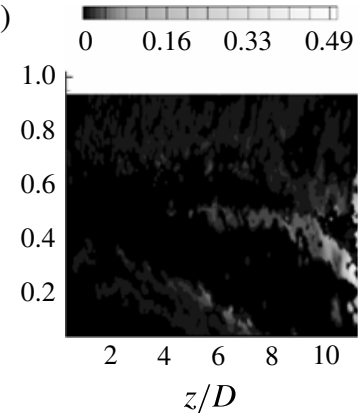

FIGURE 15. Areas of the efficient acoustic source parts that correspond to particular correlation length scale regions of the tripped jet at $S t=2.16$ and $30^{\circ}$ angle to the flow: (a) $0<L_{z}<0.03 D$, (b) $0.03 D<L_{z}<0.04 D$, (c) $0.04<L_{z}<0.05 D$, (d) $0.06 D<L_{z}<$ $0.07 D,(e) 0.07 D<L_{z}<0.09 D$ and $(f) L_{z}>0.09 D$.

a decomposition of the source field according to its axial correlation scale. In this section, we have conducted the decomposition of the noise source density $S(z, r)$, that is the integrand of (3.3a) weighted with the radius, into $N$ non-overlapping ranges $S_{i}$. Each of $S_{i}$ corresponds to a particular band of correlation length scales according to:

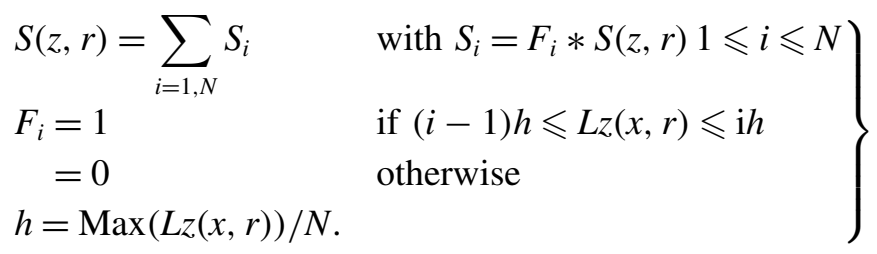

Figure 15 shows the spatial distribution of the source $S_{i}$ for several characteristic scale regions of the tripped jet case considered in figure 14(a) that is again normalized by the peak source. The calculation corresponds to the dominant vortex pairing frequency $S t=2.16$ and for noise radiated at a $30^{\circ}$ angle to the jet axis.

The dominant regions in $(a-c)$ correspond to the early shear layer region in the upstream part of the jet where vortex pairing is typically found. Those in $(d-f)$ correspond to the tail of the scale distribution and are associated with jet mixing at the end of the jet potential core $(z \sim 9 D)$. Figure 16 shows the corresponding acoustic energy budget, i.e. the energy of efficient acoustic sources that is contained in each scale band, $\mathrm{d} W / W=\int S_{i} \mathrm{~d} z \mathrm{~d} r / \int S(x, r) \mathrm{d} z \mathrm{~d} r$. The first local noise maximum is centred around $L_{z} \sim 0.04 D$ which corresponds to the peak noise source close to the location of vortex pairing in the jet. However, this is not a dominant part of the acoustic integral in (3.3a) at this frequency for the tripped jet case. The major 


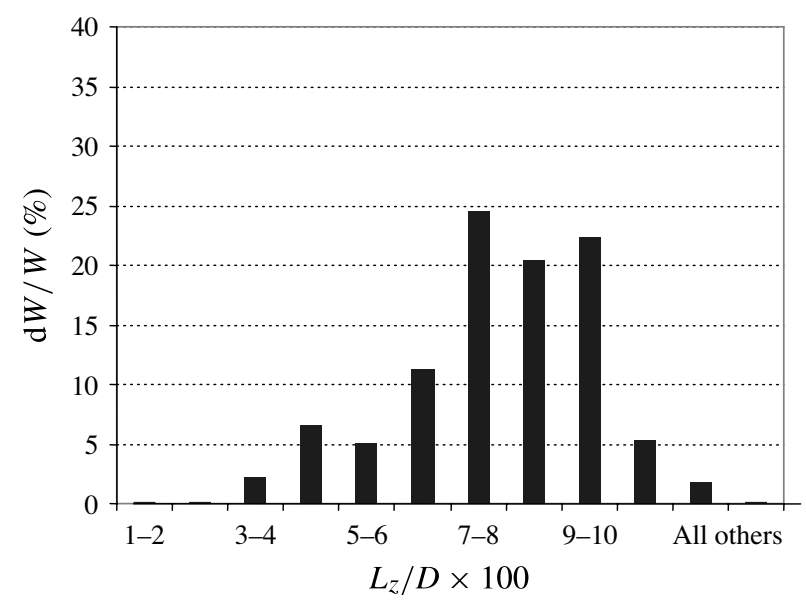

FIGURE 16. Acoustic energy budget at the vortex pairing frequency for the tripped jet case jet at $S t=2.16$ and $30^{\circ}$ angle to the flow.

mechanism contributing to the sound integral comes from the large scales that are located downstream of the end of the jet potential core $L_{z} \sim 0.07 D-0.09 D$. Although the local source density amplitudes associated with the large-scale locations are 2-3 times smaller than the peak noise associated with the vortex pairing, the area covered by the large-scales region is large, making it dominant. This suggests that the acoustic source directly associated with the vortex pairing (rather than its indirect effect on noise generation during the process of nonlinear mixing further downstream of the jet) is not the major noise mechanism at small observer angles in the case of the tripped jet.

To consider the issue of how the dominant acoustic source scales change due to the tripping of the nozzle-exit boundary layer, the source decomposition procedure, described above, is now applied to the untripped jet case.

Figure 17 shows several areas of the source $S_{i}$ for several characteristic scale regions for the untripped jet case (cf. figure 15). The calculation corresponds to the dominant vortex pairing frequency which is $S t=1.61$ in this case and $30^{\circ}$ angle to the flow.

As for the tripped jet, there appear to be the same two types of sources evident: one corresponding to the vortex pairing location that is localized in the early shear layer and the other that is located in the vicinity of the potential core of the jet, which is distributed over a larger area and can be attributed to jet mixing effects. The amplitude of the vortex pairing source in the early shear layer is a factor of 5-6 larger than the amplitude of the other noise sources. The relative difference between the two sources is notably stronger for the untripped jet, as might be expected because of the much stronger vortex pairing. We have seen previously for the tripped jet that the acoustic length scale that corresponds to the peak amplitude location of the noise source density does not correspond to the dominant part of the noise integral of $(3.3 a)$. The same is also true for the untripped jet, as shown in figure 18, which demonstrates the acoustic energy budget per scale for the untripped jet case. Similar to figure 16 for the tripped jet, for the untripped jet case figure 18 shows that it is the large acoustic scales distributed over a big jet volume downstream of the potential core of the jet that are the major contributors to noise, and not the small scales associated with the precise location of the vortex pairing event in the jet. 
(a)
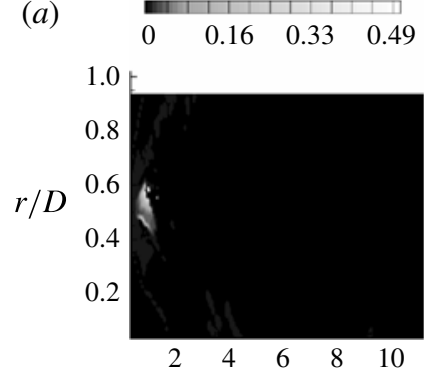

(d)

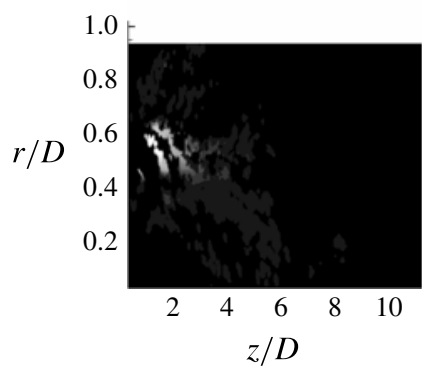

(b)

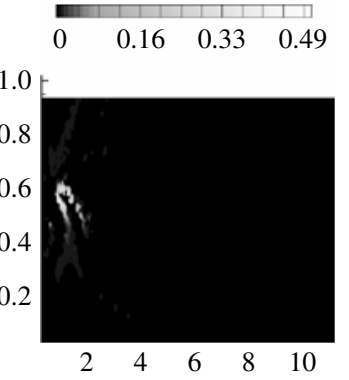

(e)
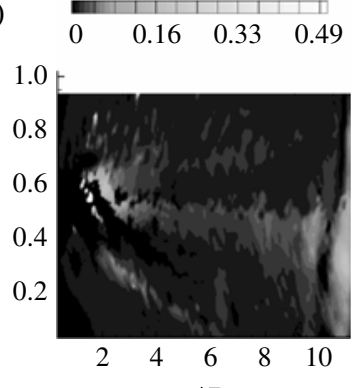

(c)
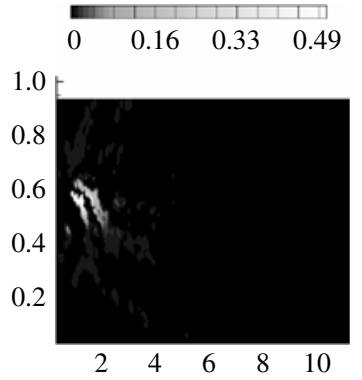

$(f)$
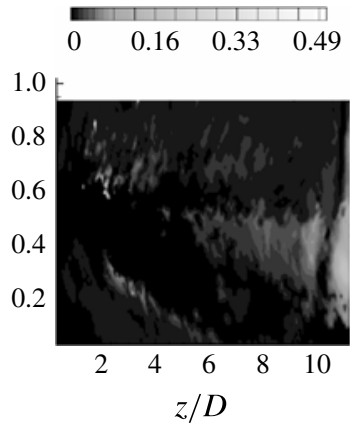

FIGURE 17. Areas of the efficient acoustic source parts that correspond to particular correlation length scale regions of the untripped jet at $S t=1.61$ and $30^{\circ}$ angle to the flow: (a) $0<L z<0.05 D$; (b) $0.05 D<L z<0.06 D$; (c) $0.06<L z<0.07 D$; (d) $0.07 D<L z<$ $0.08 D ;(e) 0.08 D<L z<0.09 D$ and $(f) L z>0.1 D$.

The comparison of figures 16 and 18 further shows that the acoustic energy distribution per correlation scale for the non-tripped case is noticeably more broadband, with at least $50 \%$ of the overall acoustic energy contributed by the large scales $L_{z}>0.1 D$. These large length scales are negligible in the acoustic energy distribution for the tripped jet case (cf. figure 16), i.e. their energy content is 3-4 orders of magnitude lower in comparison with the dominant acoustic scales. This suggests that the greater effect of large acoustic scales contributing to the noise integral obtained for the untripped jet is not an acoustic modelling artifact. Indeed, the 3-4 order difference in the acoustic energy content of the large scales between the two jets cannot be explained by a possible non-capture of the localized acoustic source corresponding to the vortex pairing location in the untripped jet since the difference between the model and the reference LES-ILEE solution reported in $\S 5$ is less than 3-4 dB.

The large-scale noise contribution of the untripped jet is generated downstream of the end of potential core locations $(z>9 D)$ where the convergence of the LES statistics was found to be less good. Thus it is this effect of the more distributed source scales for the jet with the fully laminar inflow conditions which is the most likely candidate for explaining the worse noise prediction $(3-4 \mathrm{~dB})$ in the untripped jet case in comparison with the tripped jet case $(1 \mathrm{~dB})$.

The role of the large acoustic scales in noise from initially laminar jets suggests that the effects of the 'forcing mechanism' (vortex pairing and roll-up) in the early shear layers may influence the jet and source development for some distance downstream and these 'induced' sources may dominate. 


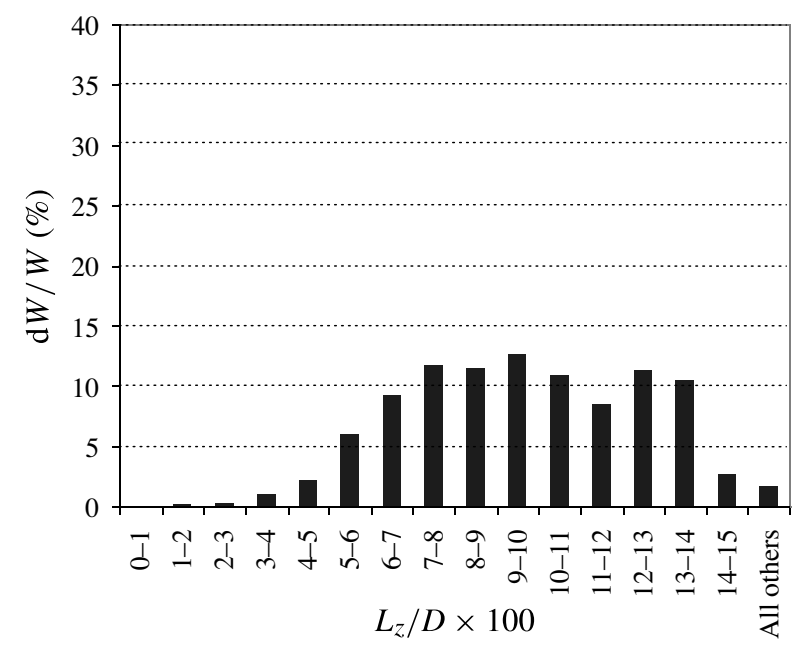

FIGURE 18. Acoustic energy budget at the vortex pairing frequency for the untripped jet case at $S t=1.61$ and $30^{\circ}$ angle to the flow.

For a useful physical interpretation of the above results that show the acoustic importance of large correlation scales at the high vortex-pairing frequency, it is useful to refer to the theoretical acoustic analogy work of Michel (2009). The Michel model can be seen as an extension of the earlier work of Michalke (1977). It starts from the classical Lighthill equation but also takes into account the effects of source interference within a simplified, distributed sound source of instability-wave type. The stochastic source nature is fully described by the coherence function $\gamma_{q}(\boldsymbol{\Delta})$ under standard statistically stationary assumptions. The coherence source function depends on the axial separation in the coherent source volume $\boldsymbol{\Delta}=\left(\Delta_{1}, \Delta_{2}, \Delta_{3}\right)$ (which can be compared with the equation for the covariance (3.5) that also includes a convecting source effect in the framework of the Goldstein acoustic analogy). The key physical parameters of the Michel model of the effective acoustic source include: (i) nondimensional length scale, $f L_{x} / v_{p}$; and (ii) non-dimensional phase speed $M_{p}=v_{p} / c_{0}$ where $f, L_{x}=\int_{-\infty}^{+\infty} \gamma_{q}\left(\Delta_{1}\right) \mathrm{d} \Delta_{1}$ and $v_{p}$ are the dimensional frequency, the integral correlation length scale and the phase speed of acoustic disturbances in the source region.

Michel shows that the best agreement between the prediction of his model for jet noise directivity and the experiments (e.g. Viswanathan 2009) is obtained when $f L_{x} \sim v_{p}$, or $L_{x} \sim \lambda M_{p}$, where $\lambda$ is the acoustic wavelength. If the phase speed is assumed to be constant for all frequencies then the length scale of the acoustic disturbances at the source will be simply proportional to the acoustic wavelength in the far field. In reality, however, in accordance with the jet instability theory of Michalke (1971), the phase speed increases with frequency, $M_{p}=M_{p}(f)$. Moreover, the phase speed is also spatially variable in a jet, being high in the early shear layers typical of the high-frequency noise generation and reduced in the developed region typical of the low-frequency noise where the jet flow decelerates. Therefore, for example, physically large structures $L_{x}$ at the source location (large correlation scales of the acoustic source in the Goldstein acoustic analogy model) can be seen as short acoustic scales $\lambda$ in the far field when the frequency and the phase speed are high. 
It may also be useful to qualitatively compare the effect that jet tripping has on reducing the size of effective acoustic sources distributed in the jet, as obtained from the acoustic source decomposition, with the effect of chevron nozzles since they also affect the noise sources by modifying the initial shear layer development. We shall do this even though there are obvious differences in inflow boundary conditions at the nozzle exit between the initially laminar jets considered and a fully turbulent high -Reynolds-number jet from a typical engine exhaust.

Chevron nozzles reduce noise at small angles to the jet in comparison with axisymmetric jets but can also lead to noise increase for large angles (e.g. Xia, Tucker \& Eastwood 2009). A possible mechanism of noise reduction at small angles is associated with large-scale flow mixing generated by the chevrons that leads to a thickening of the shear layer and slows down the growth of instability waves, which, in turn, is thought to determine noise generation of high-speed subsonic jets at small angles to the jet (Tam et al. 2008). In comparison with the large-scale mixing typical of chevron jets, jet tripping reduces noise both for small and large angles to the jet for the cases studied and does so without a notable thickening of the jet shear layer or potential core contraction. The jet tripping affects small flow scales within the early jet shear layers and leads to a more gradual jet development, which, in turn, leads to a longer potential core length. Despite the extended potential core of the tripped jet, as the acoustic post-processing showed, the correlation length of the effective acoustic source, i.e. the length scale which mostly contributes to the noise integral of $(3.3 a)$, is reduced in comparison with that for the untripped jet and it is this that determines the noise reduction.

Finally, the effect of observer angle on the acoustic energy budget for the tripped jet case is investigated. As well as exhibiting inflow boundary conditions more typical of the high-speed jets more commonly used in experiments, this case corresponds to the best agreement between the acoustic analogy model and the reference LES-ILEE solution (within $1 \mathrm{~dB}$ for both $30^{\circ}$ and $90^{\circ}$ angle to the jet). We will use this agreement as a justification that the model assumptions such as the convergence of the statistical source model, the Gaussian nature of the correlation functions and the approximation of the three spatial scales by a single axial correlation scale were reasonably accurate in this case.

There has been some debate in the literature about the directivity of high-speed subsonic jet noise: how the highly directional behaviour observed in the far-field sound microphone measurements relates to the scales within the jet. For example, in accordance with one popular approach (Tam \& Auriault 1999) there are two distinct source mechanisms of jet noise. One corresponds to large-scale coherent structures that are associated with peak noise propagating at small angles to the jet. The other source is attributed to the fine-scale turbulence that exerts effective turbulent pressure on its surroundings and is acoustically compact, omni-directional, random and spatially uncorrelated (Tam et al. 2008, p. 23).

Because of the LES data limitations discussed previously, the lowest frequency the present acoustic analogy model can accurately capture is about $S t=0.5$, with perhaps qualitative agreement at $S t=0.3$. On the other hand, as we have seen previously, the LES data show significant source correlations over axial distances of $0.1 D-0.2 D$. Therefore, despite the limited accuracy at low frequencies and in accordance with the fine-scale/large-scale noise theory, one can expect that there is a significant part of the large-scale noise source that remains in the current data. This part of the large-scale noise source should have a very different directivity in comparison with the uncorrelated fine-scale noise. We next try to detect the two different acoustic source mechanisms for the tripped jet case by examination of the decomposed sources. 

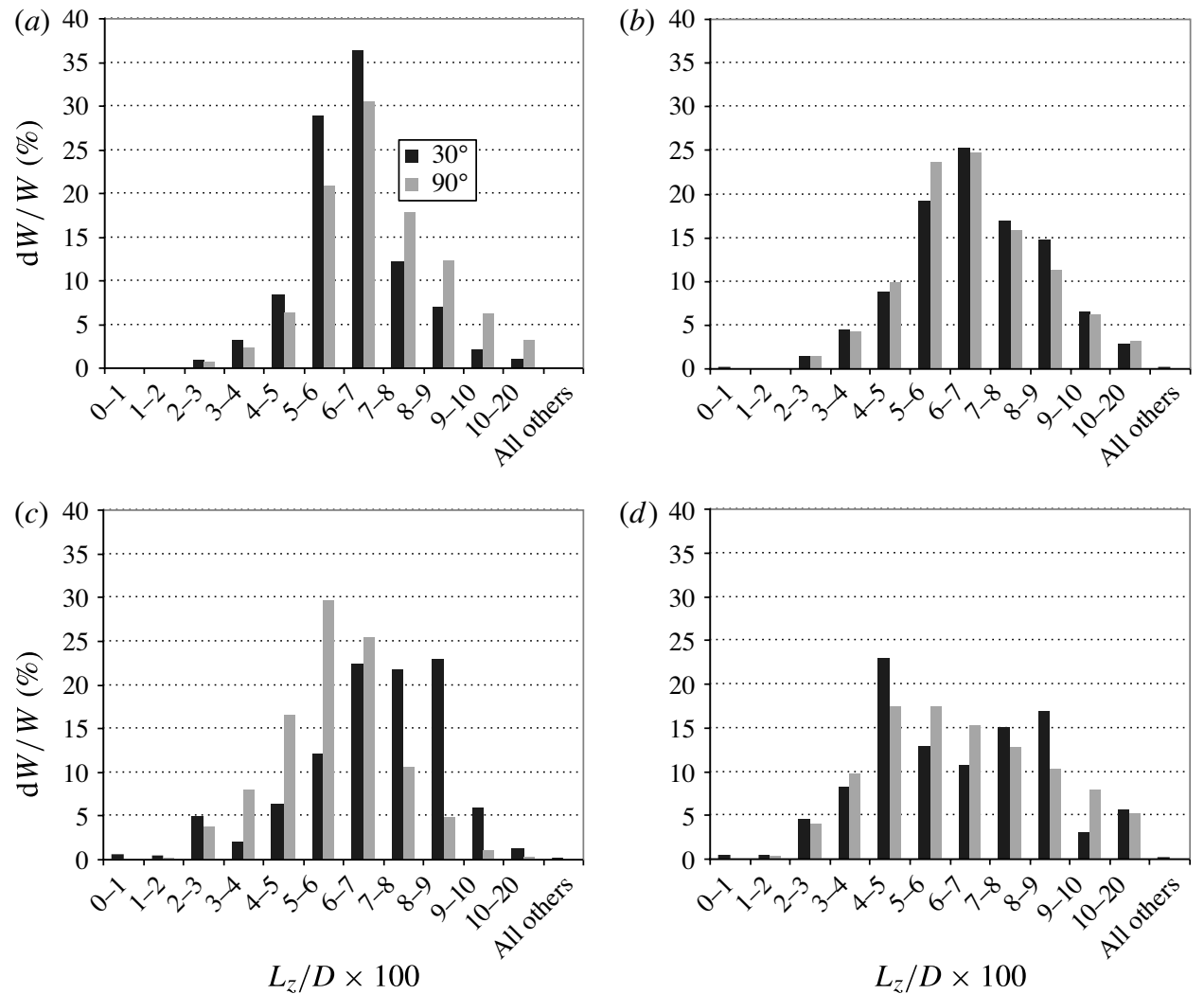

FIGURE 19. Acoustic energy budget for the tripped jet case at: (a) $S t=0.3 ;(b) S t=1$; (c) $S t=3 ;(d) S t=6$.

The source decomposition technique (6.1) is applied for observer angles of $30^{\circ}$ and $90^{\circ}$ to the jet. The frequency range considered, $0.3<S t<6$, corresponds to the range of good agreement between the predictions of the acoustic analogy method and the reference LES-ILEE simulation. Figure 19 shows the corresponding acoustic energy budgets.

For the low-frequency case, $S t=0.3$, the acoustic energy distribution at $90^{\circ}$ has significant contributions from larger scales (figure 19a) when compared with the more peaky small-angle case. As frequency is increased, there is a gradual broadening of the contributing range of scales for both angles. For the $30^{\circ}$ case, at $S t=3$, the peak density of the acoustic source is be found in 20-30\% longer scales when compared to the $90^{\circ}$ angle. The distributions for $90^{\circ}$ generally have a single-maximum symmetric profile, with that for high frequencies tending to be centred at the scales close to those of vortex pairing, $L_{z} \sim 0.04 D$. In contrast, for $30^{\circ}$ a second maximum emerges in the energy distribution. The latter corresponds to the large-scale structures at the end of the jet potential core of characteristic size $L_{z} \sim 0.07 D-0.09 D$. Despite these differences, the source analysis performed has not revealed any significant dependence on frequency and observer angle of the source length scales, certainly no tendency for short scales to dominate at high frequency and high angle. Admittedly, this could be the result of limited data available to us in this study and extending the present acoustic modelling to other jet cases will be the subject of our future work. 


\section{Conclusion}

A modified Goldstein acoustic analogy method based on a statistical equivalent source model obtained from large eddy simulation (LES) data, with a full capture of sound-mean flow propagation effects by solving the linearized Euler equations, is implemented for high-Reynolds-number initially laminar jets corresponding to different inflow boundary conditions: tripped and untripped inflow cases. Post-processing of the LES data is performed and examined critically, providing conservative estimates of the areas of the jet which are amenable to analysis using the present acoustic analogy model. These areas are the main areas of noise generation in the jet potential core including the jet edges and the outflow boundary regions just downstream of the end of the potential core.

An attempt was made to fit a Gaussian form to fourth-order correlation statistics and was successful for most of the area of the jet for which LES statistics were available. Based on previous experience, post-processing of the LES fields shows that the credibility limit for the acoustic analogy model predictions at low frequencies is St $\sim 0.2-1$.

For both jets, the distribution of correlation source scales is obtained. For the lip-line location, the scales in three spatial directions have similar relative amplitudes to those found experimentally by Morris \& Zaman (2010).

The sound power spectral density predictions of the acoustic analogy model agree with the reference large eddy simulation-isotropic linearized Euler equations (LES-ILEE) method to within $1 \mathrm{~dB}$ for the tripped jet, and within 3-4 $\mathrm{dB}$ for the untripped jet, for $30^{\circ}$ and $90^{\circ}$ observer angles to the jet and the frequency range $0.8<S t<6$. The less good agreement for the untripped jet is associated with strong vortex pairing located in the outer shear layers. The discrepancy at high frequencies, $S t>6$, between the two methods is likely to be caused by LES-ILEE surface resolution issues and those for low frequencies, $S t<0.5-0.8$, by the spurious low-frequency noise reported for the original LES-ILEE simulation. For the tripped jet case, the importance of the complete anisotropic statistical source description, and the full propagation model based on linearized Euler equations, is demonstrated. In particular, for small angles to the jet, the use of a simplified locally parallel jet flow model when accounting for mean flow-sound interaction effects can lead to up to an $8 \mathrm{~dB}$ error in sound pressure levels when compared with the full linearized Euler solution. The error due to the non-compact-source-scales approximation is found to be less severe.

The peak location of noise source density was investigated for tripped and untripped jets for a few frequencies. The calculation of local source densities averaged over different jet areas shows that the effective acoustic source for the untripped jet has a smaller source contribution from the last $20 \%$ of the axial extent of jet in comparison with the tripped case. It has been argued that a possible explanation for this effect is that the acoustic sources for the untripped jet are distributed over a large axial extent and have a richer energy content in the downstream part of the jet when compared with the tripped jet. The full source axial extent may not be fully captured due to the limitations of the axial extent of the statistical source data available. This explanation is consistent with the results of a detailed analysis of the effective sound sources in each jet case that showed a significantly more broadband acoustic source distribution for the untripped jet case, with some $50 \%$ of the acoustic energy contained in the jet locations susceptible to the lack of convergence of the LES data. For an observer angle of $30^{\circ}$ to the jet downstream direction, when focusing on the vortex pairing frequency, there are localized sources identified both for the tripped and untripped jets in the 
early shear layer locations. These locations are close to those reported as exhibiting vortex pairing by Bogey \& Bailly (2010).

A numerical source decomposition technique based on the fourth-order correlation length scales has been used for a detailed investigation of jet noise sources in the tripped jet as a way of attributing sound to different length scales in the jet. For the vortex pairing frequency, there are two types of noise source found. One, which corresponds to the peak source amplitude, is located at the upstream end of the jet and is associated directly with vortex pairing. The other, which is the dominant source in the acoustic integral, is associated with jet mixing at the end of the jet potential core. This acoustic source imaging technique is used to compare the effective source scale contributions for the tripped and untripped jet cases. It is shown that nozzle boundary layer tripping leads to a reduction in the contribution of large acoustic scales to the noise integral. This effect is discussed and qualitatively compared with the effect of chevron nozzles that reduce noise at small angles to the jet flow, thickening the jet shear layer and reducing the jet mixing area (the potential core length). When compared with the large-scale mixing typical of chevron jets, tripping reduces noise both for small and large angles to the jet without a notable thickening of the jet shear layer or potential core contraction. Its major effect on noise reduction is seen as a reduction in the dominant correlation length of the acoustic source.

The results obtained in this paper regarding the acoustic importance of large scales at the vortex-pairing frequency, which is high for the jets with thin shear layers considered, are discussed in the light of the acoustic analogy modelling work of Michalke (1977) and Michel (2009). Following Michel, it is argued that physically short scales may play the role of large structures for high frequencies when judged by their influence on the sound integral.

For the tripped jet that is more typical of those which are usually used in experiments, the acoustic energy budget is computed as a source distribution per correlation scale band for a wide range of frequencies for radiation at $30^{\circ}$ and $90^{\circ}$ observer angles. A broadening of energy distribution with frequency is observed. No significant energy scale separation depending on the observer angle, indicative of the presence of two distinctive noise sources, one highly directional and correlated and the other omni-directional and uncorrelated, is found. Admittedly, this could be the result of limited data available to us in this study, and extending the present acoustic modelling to other jet cases will be the subject of our future work.

\section{Acknowledgements}

The work of S.K. was supported by the Royal Society of London. The authors are grateful to S. Hale for his help in LES data post-processing in the course of a Summer University Research Opportunity Project in Cambridge.

\section{Appendix. Methodology of fitting the fourth-order velocity correlation coefficients to an analytical decaying Gaussian model}

The calculation of the scales in the axial direction is considered first, which corresponds to letting $\Delta_{1}=\mathrm{d} x, \Delta_{2}=\Delta_{3}=0$ in (3.5). A four-step procedure for computing the correlation fits is used as follows.

(i) Local maxima for the Gaussian are calculated from the numerical $R_{i j k l}(y, \mathrm{~d} x, \mathrm{~d} t)$; if the local maxima are not positive the correlation fit is flagged as failing.

(ii) Assuming the magnitude of the correlation peak decays with spatial separation, the numerical value which is approximately $60 \%$ of the magnitude of the 

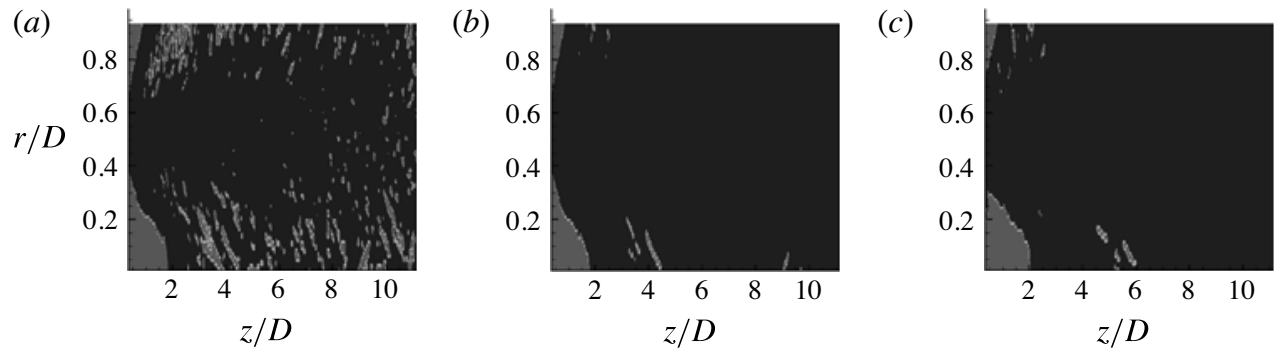

FIGURE 20. Improving the statistical convergence of LES data with a bigger number of time samples; areas of the domain where the correlation fit works are shown dark (blue online), and when it fails are lighter (red online): (a) $50 \%$ of time samples and (b) $100 \%$ of time samples for the untripped jet; $(c) 100 \%$ of time samples for the tripped jet.

autocorrelation amplitude $R_{i j k l}^{(60 \% p e a k)} \sim 0.6 A_{i j k l}(\boldsymbol{y})$ and its corresponding separation in time $\tau_{\text {peak }}=\mathrm{d} t=\Delta_{1} / \tilde{v}_{1}$ are considered, and the correlation time based on the $60 \%$ values is computed $\tau_{s}(\boldsymbol{y})=\tau_{\text {peak }} / \log \left(A_{i j k l}(\boldsymbol{y}) / R_{i j k l}^{(60 \% p e a k)}\right)$; if this correlation time is not positive for positive spatial separations the correlation fit is flagged as failing.

(iii) The correlation velocity based on the local $60 \%$ peak values is computed, $\tilde{v}_{1}=\Delta_{1} / \mathrm{d} t$; from the characteristic width $\Delta t^{(\text {width })}$ of the $60 \%$ profile that corresponds to the amplitude decay from $R_{i j k l}^{(60 \% \text { peak })}=R_{i j k l}^{(60 \% \text { peak })}(0)$ to $R_{i j k l}^{(60 \% \text { peak })}\left(\Delta t^{(\text {width })}\right)$ the length scale is computed: $l_{s z}(\boldsymbol{y})=\sqrt{\log (2)\left(\tilde{v}_{1} \Delta t^{(\text {width })}\right)^{2} / \log \left(R_{i j k l}^{(60 \% p e a k)}(0) / R_{i j k l}^{(60 \% p e a k)}\left(\Delta t^{(\text {width })}\right)\right)}$.

Once the axial correlation values are calculated they are substituted into $(3.3 a)$ and the correlation lengths in the remaining two spatial directions are calculated in a similar manner.

The above Gaussian fitting procedure has been applied to the data for the tripped and untripped jets. Figure 20 shows that, as the period of time averaging used for the computation of fourth-order correlations increases, a greater area of the jet satisfies the Gaussian fit criterion outlined above. In terms of the statistical convergence of LES data, this suggests that, in the sense of fourth-order correlations, the convergence of the present LES data improves when there are more time samples taken for averaging.

Figure 21 shows how well (except for some discrepancies at vanishingly small time delays $\tau \rightarrow 0$ with zero spatial separation, $\Delta=0$, noted earlier) the Gaussian model for the two-point two-time fourth-order correlations works in the jet shear layer locations $(z=4 D, r=0.5 D)$. Previous work by Karabasov et al. (2010) showed that the decaying Gaussian-type correlation function is also in a good agreement with the results of jet experiments.

As the distance from the nozzle exit increases, the shape of the correlation function starts to deviate from the Gaussian shape. For example, the correlation function for large space-time separations does not tend to zero but tends to a constant value which is $\sim 10-20 \%$ of the peak correlation amplitude for $z \sim 10 D-12 D$ for both jet cases. The same trend is also observed for the outer radial jet locations, $r \sim 0.8 D-1 D$.

The stagnation of the numerical correlation function to a non-zero value for large separations is not physical, indicating that for such locations the LES data are less well converged. The main effect of the lack of convergence observed is expected to be 

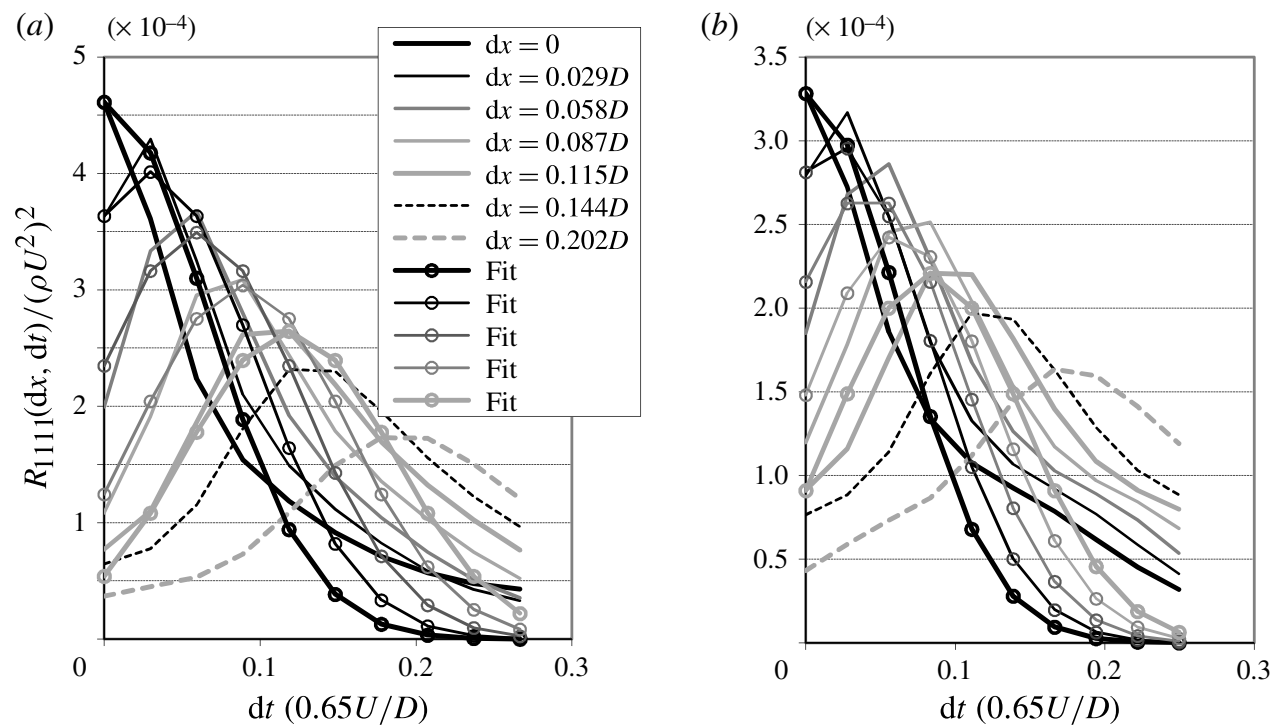

FIGURE 21. Correlation coefficients and Gaussian fits for the tripped jet in the shear layer location $r=0.5 D$ at $(a) z=4 D$ and $(b) z=8 D$.

on the low-frequency part of the noise spectrum because it is the low-frequency sound that corresponds to the large separation times of the fourth-order correlations in the acoustic analogy source.

In addition to large axial and radial locations, another challenging jet region for the Gaussian correlation fits is close to the inner edge of the shear layer. This region, despite a relatively small correlation amplitude, is distributed over a large area, hence can be potentially important for the noise integral. In these locations the shear layer protrudes intermittently into the jet potential core which creates regions of large differences in scales over a relatively small area in the jet. Figure 22 shows examples of correlation coefficients in two jet locations where the correlation lengths vary by a factor of 2-3 over two jet diameters, as does the correlation time. Interestingly, the Gaussian-fit model works rather well for both these jet locations, confirming the model robustness.

It should be noted that the correlation functions presented in figures 21 and 22 do not show any significant negative regions because of the insufficiently long time delay available for these regions from the LES post-processed data. For longer time delays, negative portions of the decaying correlation function are expected to appear, in accordance with the experimental evidence (e.g. Morris \& Zaman 2010) and the previous calculations (Karabasov et al. 2010).

Finally, there are still locations in the jet where the Gaussian fit fails even when using the full $100 \%$ of the time samples available. These misfits fall into two categories, as illustrated in figure 23. The data shown correspond to the untripped case but are qualitatively similar to those of the tripped jet case. The first category is most typical of the upstream parts of the jet and also of the outer side of the shear layer $(a)$. Most of these locations disappear when the number of time samples is increased from $50 \%$ to $100 \%$, suggesting a probable lack of numerical-LES-data convergence. The second category is typically in the region towards the end of the 

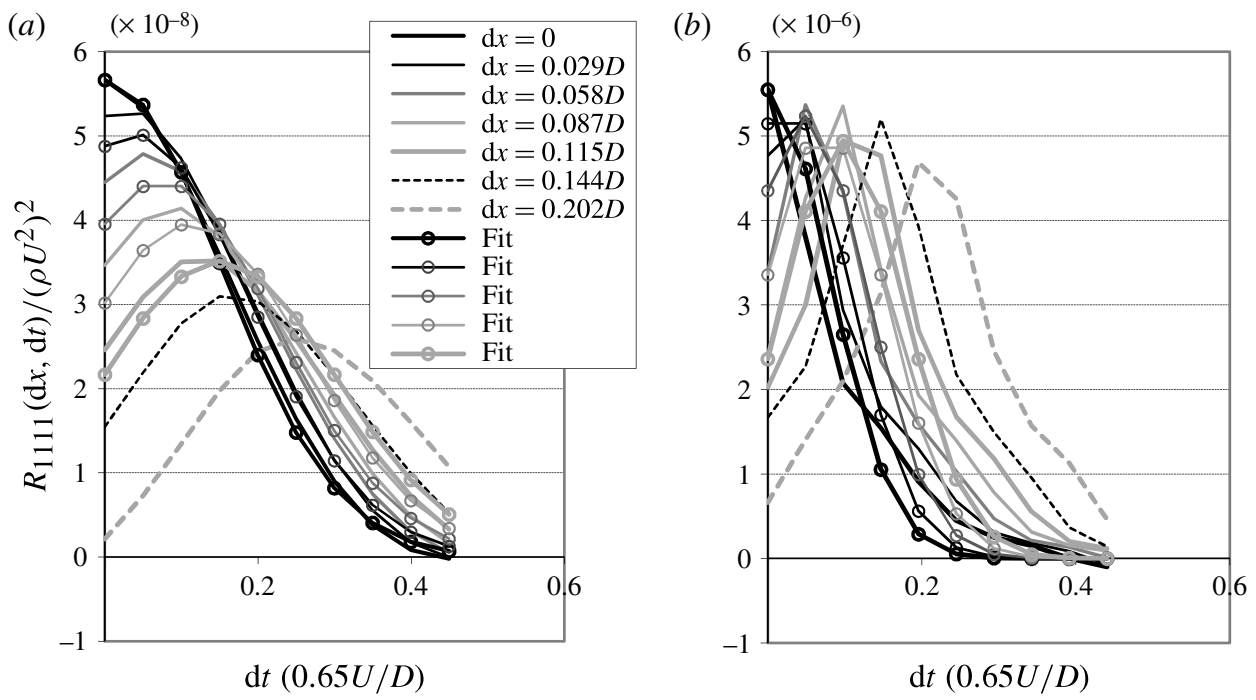

FIgURE 22. Correlation coefficients and Gaussian fits in the tripped jet at the location $r=0.1 D:(a) z=3 D$ and $(b) z=5 D$.

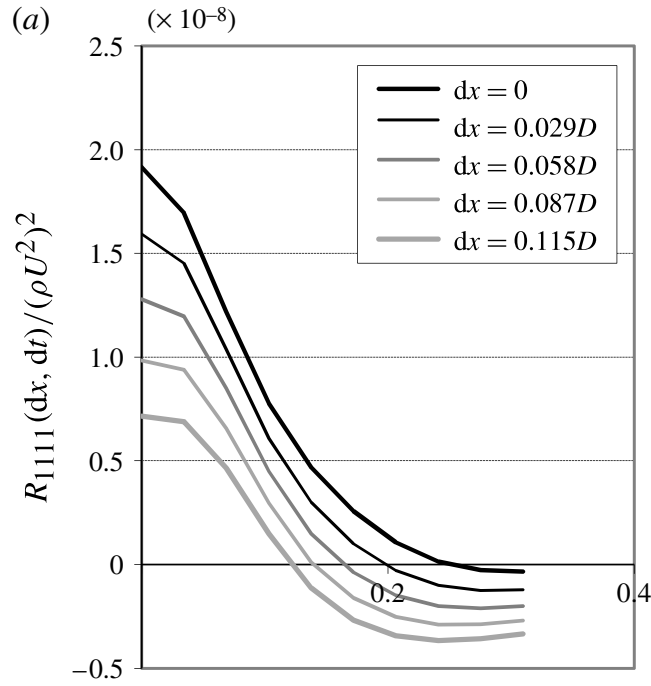

$\mathrm{d} t(0.65 U / D)$

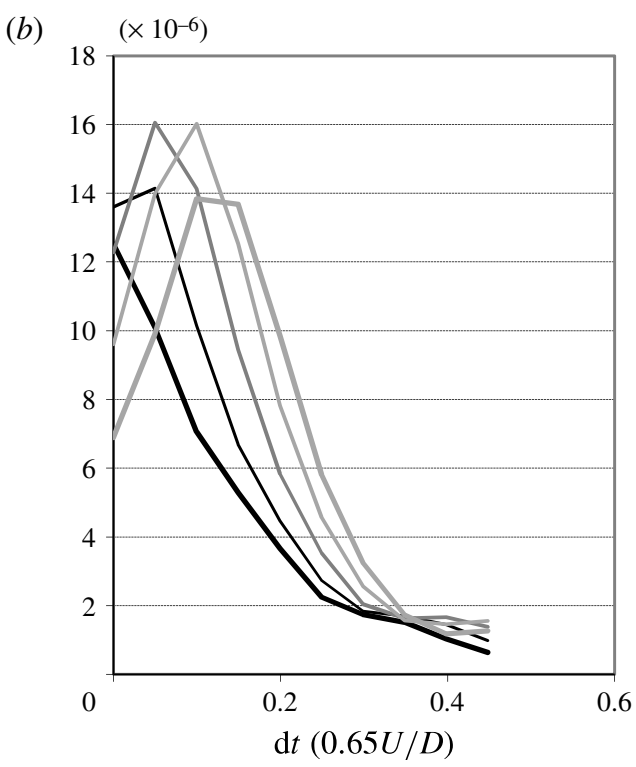

FIGURE 23. Typical cases of the fourth-order correlation coefficients not well fitted by a decaying Gaussian shape: $(a)$ in the shear layer location at $z=0.5 D$; and $(b)$ at $r=0.1 D$ and $z=4 D .100 \%$ LES data time samples are used.

potential core and on the inner side of the developed shear layer as it intrudes into the jet core. This can be associated with growing and decaying waves $(b)$ and most of these locations survive the transition from $50 \%$ to $100 \%$ data sampling. 
The misfits lead to some areas of the jet field being excluded from the model which in turn leads to some underestimation of the noise integral. In order to quantify this effect, the amplitudes of the correlation coefficients in the 'misfit' jet locations are computed and compared with the typical correlation peak values in the locations where the Gaussian model works. This model self-consistency check showed that the locations where the Gaussian fit fails are typically 2-5 orders of magnitude smaller than the peak correlation amplitudes in the jet shear layer, confirming that the Gaussian model approximates the fourth-order statistics of the LES data of Bogey \& Bailly (2010) reasonably well.

\section{REFERENCES}

AfSAR, M. Z. 2010 Asymptotic properties of the overall sound pressure level of sub-sonic air jets using isotropy as a paradigm. J. Fluid Mech. 664, 510-539.

Bogey, C. \& BAILly, C. 2005 Effects of inflow conditions and forcing on a Mach 0.9 jet and its radiated noise. AIAA J. 43 (5), 1000-1007.

Bogey, C. \& BAilly, C. 2009 Turbulence and energy budget in a self-preserving round jet: direct evaluation using large-eddy simulation. J. Fluid Mech. 627, 129-160.

Bogey, C. \& BAILly, C. 2010 Influence of nozzle-exit boundary-layer conditions on the flow and acoustic fields of initially laminar jets. J. Fluid Mech. 663, 507-540.

Bogey, C., Marsden, O. \& BAilly, C. $2011 a$ Large-eddy simulation of the flow and acoustic fields of a Reynolds number $10 \mathrm{e} 5$ subsonic jet with tripped exit boundary layers. Phys. Fluids 23 (3), 035104.

Bogey, C., Marsden, O. \& Bailly, C. $2011 b$ Influence of initial turbulence level on the flow and sound fields of a subsonic jet at a diameter-based Reynolds number of 10e5. J. Fluid Mech. (submitted). See also AIAA Paper 2011-2837.

BRIdges, J. E. \& Hussain, A. K. M. F. 1987 Roles of initial conditions and vortex pairing in jet noise. J. Sound Vib. 117 (2), 289-311.

Colonius, T. \& LelE, S. K. 2004 Computational aeroacoustics: progress on nonlinear problems of sound generation. Prog. Aerosp. Sci. 40, 345-416.

Crighton, D. G. 1981 Acoustics as a branch of fluid mechanics. J. Fluid Mech. 106, 261-298.

Ffowcs Williams, J. E. 1963 The noise from turbulence convected at high speed. Phil. Trans. R. Soc. Lond. A 255, 469-503.

Goldstein, M. E. 2002 A unified approach to some recent developments in jet noise theory. Intl J. Aeroacoust. 1 (1), 1-16.

Goldstein, M. E. 2003 A generalized acoustic analogy. J. Fluid Mech. 488, 315-333.

Goldstein, M. E. 2011 Recent developments in the application of the generalized acoustic analogy to jet noise prediction. Intl J. Aeroacoust. 10 (2,3), 89-116.

Goldstein, M. E. \& LEIB, S. J. 2008 The aero-acoustics of slowly diverging supersonic jets. J. Fluid Mech. 600, 291-337.

Goldstein, M. E. \& Rosenbaum, B. 1973 Effect of anisotropic turbulence on aerodynamic noise. J. Acoust. Soc. Am. 54, 630-645.

GROSCHE, F.-R. 1974 Distributions of sound source intensities in subsonic and supersonic jets. AGARD-CP-131, 4-1-4-10. AGARD.

Howe, M. S. 2003 Theory of Vortex Sound. Cambridge University Press.

Hill, W. G., Jenkins, R. C. \& Gilbert, B. L. 1976 Effects of the initial boundary-layer state on turbulent jet mixing. AIAA J. 14 (11), 1513-1514.

Karabasov, S. A. 2010 Understanding jet noise. Phil. Trans. R. Soc. Lond. A 368, 3593-3608.

Karabasov, S. A., Afsar, M. Z., Hynes, T. P., Dowling, A. P., McMullan, W. A., Pokora, C. D., PAge, G. J. \& McGuirk, J. J. 2010 Jet noise: acoustic analogy informed by large eddy simulation. AIAA J. 48 (7), 1312-1325.

Karabasov, S. A., Bogey, C. \& Hynes, T. P. 2011 Computation of the noise of initially laminar jets using a statistical approach for the acoustic analogy: application and discussion. AIAA Paper 2011-2929. 
Karabasov, S. A. \& Hynes, T. P. 2006 Adjoint linearized Euler solver in the frequency domain for jet noise modelling. AIAA Paper 2006-2673.

Khavaran, A., Bridges, J. \& Georgiadis, N. 2005 Prediction of turbulence-generated noise in unheated jets. NASA TM 2005-213827.

Lighthill, M. J. 1952 On sound generated aerodynamically: I. General theory. Proc. R. Soc. Lond. A 222, 564-587.

Lilley, G. M. 1958 On the noise from air jets. Aeronaut. Res. Counc. $R \&$ \& 20, 376.

LILlEy, G. M. 1972 Generation of sound in a mixing region, Lockheed Aircraft Co. 4th Monthly progress report on contract F-33615-71-C-1663, Marietta.

Maestrello, L. \& MCDAid, E. 1971 Acoustic characteristics of a high-subsonic jet. AIAA J. 9 (6), 1058-1066.

MichalKe, A. 1971 Instabilität eines kompressiblem runden Freistrahls unter Berücksichtigung des Einflusses der Strahlgrenzschichtdicke. Z. Flugwiss. 19, 319-328 English Translation: NASA TM 75190 (1977).

Michalke, A. 1977 On the effect of spatial source coherence on the radiation of jet noise. $J$. Sound Vib. 55, 377-394.

MicheL, U. 2009 The role of source interference in jet noise. In AIAA Paper 2009-3377.

MORRIS, P. J. 2009 A note on noise generation by large-scale turbulent structures in subsonic and supersonic jets. Intl J. Aeroacoust. 8 (4), 301-316.

MORRIS, P. J. \& FARASSAT, F. 2002 Acoustic analogy and alternative theories for jet noise prediction. AIAA J. 40, 671-680.

MorRIS, P. J. \& ZAMAN, K. B. M. Q. 2010 Velocity measurements in jets with application to noise source modelling. J. Sound Vib. 329 (4), 394-414.

Power, O., Kerherve, F., FitzPatrick, J. \& Jordan, P. 2004 Measurements of turbulence statistics in high subsonic jets. AIAA Paper 2004-3021.

Ribner, H. S. 1964 The generation of sound by turbulent jets. Adv. Appl. Mech. 8, 108-182.

Samanta, A., Freund, J. B., Wei, M. \& Lele, S. K. 2006 Robustness of acoustic analogies for predicting mixing-layer noise. AIAA J. 44, 2780-2786.

STANLEy, S. A. \& SARKAR, S. 2000 Influence of nozzle conditions and discrete forcing on turbulent planar jets. AIAA J. 38 (9), 1615-1623.

TAM, C. K. W. \& Auriault, L. 1998 Mean flow refraction effects on sound from localized sources in a jet. J. Fluid Mech. 370, 149-174.

TAM, C. K. W. \& AUriault, L. 1999 Jet mixing noise from fine scale turbulence. AIAA J. 22 (2), $145-153$.

TAM, C. K. W., Golebiowski, M. \& Seiner, J. M. 1996 Two components of turbulent mixing noise from supersonic jets. AIAA Paper 96-1716.

Tam, C. K. W., Viswanathan, K., Ahuja, K. K. \& Panda, J. 2008 The sources of jet noise: experimental evidence. J. Fluid Mech. 615, 253-992.

Tester, B. J. \& Morfey, C. L. 1976 Development in jet noise modelling - Theoretical predictions and comparisons with measured data. J. Sound Vib. 46, 79-103.

ViswanAthan, K. 2009 Mechanisms of jet noise generation: classical theories and recent developments. Intl J. Aeroacoust. 8 (4), 355-407.

Xia, H., Tucker, P. G. \& EASTwood, S. 2009 Large-eddy simulations of chevron jet flows with noise predictions. Intl J. Heat Fluid Flow 30 (1), 1067-1079.

ZAMAN, K. B. M. Q. $1985 a$ Effect of initial condition on subsonic jet noise. AIAA J. 23, $1370-1373$.

ZAMAN, K. B. M. Q. $1985 b$ Far-field noise of subsonic jet under controlled excitation. J. Fluid Mech. 152, 83-111.

ZAMAN, K. B. M. Q. 2011 Effect of nozzle exit conditions on subsonic jet noise, AIAA Paper 2011-2704.

Zaman, K. B. M. Q. \& Hussain, A. K. M. F. 1980 Vortex pairing in a circular jet under controlled excitation. Part 1. General jet response. J. Fluid Mech. 101 (3), 449-491. 